\title{
Three denials of time in the interpretation of canonical gravity
}

\author{
Karim P. Y. Thébault ${ }^{1}$ \\ Centre for Time, Department of Philosophy, University of Sydney
}

\begin{abstract}
The analysis of the temporal structure of canonical general relativity and the connected interpretational questions with regard to the role of time within the theory both rest upon the need to respect the fundamentally dual role of the Hamiltonian constraints found within the formalism. Any consistent philosophical approach towards the theory must pay dues to the role of these constrains in both generating dynamics, in the context of phase space, and generating unphysical symmetry transformations, in the context of a hypersurface embedded within a solution. A first denial of time in the context of a position of reductive temporal relationalism can be shown to be troubled by failure on the first count, and second denial in the context Machian temporal relationalism can be found to be hampered by failure on the second. A third denial of time, consistent with both the of the Hamiltonian constraints roles, is constituted by the implementation of a scheme for constructing observables in terms of correlations. The motivation for and implications of each of these three denials are investigated.
\end{abstract}

Keywords: Problem of Time, Canonical Gravity, Constraints, Symmetry, Observables, Relationalism

\section{Introduction}

That the boundary between physics and philosophy is often found to be a vague one should perhaps not surprise us given the overlapping concerns of the two disciplines. Yet that these concerns may be not just overlapping but entangled, and that the boundary may be not just vague but illusory would, if it were to prove to be the case, be highly non-trivial. It is within this controversial and treacherous border-lands, where physics meets philosophy, that the deniers of time may conspire with the interpreters of general relativity. In this paper I will investigate the extent to which the particular physico-mathematical formalism that is constituted by the canonical formulation of general relativity forces upon

\footnotetext{
${ }^{1}$ karim.thebault@gmail.com
} 
us a fundamental denial of temporality. In particular, I will examine three approaches towards canonical gravity that underpin three distinct senses in which the theory may be interpreted as timeless. In each case a stance as to how to understand certain aspects of the formalism shall be shown to be intwined with a philosophical position as to the nature of time; and in each case we shall find that in addition to the physical formalism motivating a philosophical position, the relevant interpretative considerations may be seen to motivate a position with regard to the formalism. Our concern here will not be with broader questions as to how physical theory and its interpretation should be understand to relate to ontological assertions in either in general, nor with regard to the specific case of time. Rather, we shall restrict ourselves to how the interpretation and formalism in question is related to temporality and its denial, specifically and solely with regard to general relativity. As general relativity is at the moment the only physical theory which can acutely describe the behaviour of clocks there is of course reasonable grounds to assert that whatever this theory says about time, is precisely what time is, simpliciter. However, we will not here venture in to discussing the philosophical subtitles of such strong ontological claims relating to either time or its denial. When we talk of denying time, we will only do so in the sense of asserting that a particular weakened notion of temporality is associated with the particular interpretation of the physical theory in question.

Our first denial of time will be predicated upon passage to formalism within which only static universes may be represented. Thus, it involves an interpretation of general relativity in which there is no change because there is no dynamical structure at all. This denial is in part motivated by application of the standard machinery for dealing with the Hamiltonian formulation of a theory displaying gauge symmetry, and in part by a desire to implement a reductive notion of spacetime relationalism specifically within the Hamiltonian formulation of general relativity which is constituted by the canonical version of the theory. Ultimately it shall be found to rest on a misunderstanding of the extent to which canonical general relativity can be treated as a standard gauge theory. Although this approach will lead to a formalism amenable to the philosophical goals which at least partially motivate it, we shall find that a deficiency in dynamical structure indicates this formalism is fundamentally inadequate for representing the world. Thus, both the basis for and implications of the first of our denials will themselves be denied.

As well as differing from the first in terms of the formal moves involved, the second of our denials differs as to the philosophical stance with regard to time that it motivates, and that it is motivated by. In this context the denial of time amounts to a far weaker assertion that temporal structure has a secondary, derived nature - being constituted parasitically upon relations. The fundamental structure of our ontology is taken to be spatial in nature and in accordance with a Machian notion of dynamics, time is seen to emerge equitably and uniquely within the formalism. The viability of this approach will be found to rest upon what methodology can be found to respond to a problem of indeterminism which is generic within gauge theory and is, in fact, one of aspect of the motivation for our erroneous first denial. Two responses shall be 
considered, but only the second, which involves a radical adjustment to the role of absolute scale within general relativity, will be found to be consistent with the philosophical framework for denying time with which we are concerned.

Our third and final denial of time will be found to be both much more tenable than the first and much stronger than the second. The capacity to represent dynamical universes will be retained and yet the notions of both time and change will be dispensed with. Under this interpretation the ontology of the theory consists of correlations which are effectively smeared non-locally across entire histories. However, since these perennials come in families they may collectively be used to represent worlds which have dynamical structure beyond the trivial. Interestingly, although this denial does correspond to a fundamental denial of change in a Paramenadian sense, it allows us to maintain exactly the fundamental four-dimensionality of ontology that is dispensed with in our second, arguably weaker, denial. Before we embark on the main body of our discussion we must first consider carefully canonical general relativity by itself, both with regard to its formal structure and its relationship with the original covariant formulation of the theory.

\section{General Relativity and The Problem of Time}

\subsection{The Canonical Theory}

Consider the covariant formulation of Einstein's general theory of relativity (in vacuo) according to the Einstein-Hilbert action:

$$
S=\frac{1}{\kappa} \int_{\mathcal{M}} d^{4} x \sqrt{|\operatorname{det}(g)|} R=\int_{\mathcal{M}} d^{4} x \mathcal{L}_{E H}
$$

where $\mathcal{M}$ is a four dimensional manifold which we assume to be spatially compact without boundary and have arbitrary topology, $g_{\mu \nu}$ is a metric tensor field of Lorentzian signature and $R$ is the Ricci scalar. Variation of this action according to a least action principle leads to the Einstein field equations the solution of which leads to an expression for the metric tensor. This tensor equips the manifold $\mathcal{M}$ with a geometry and thus we arrive at the set of four geometries, $\left(M, g_{\mu \nu}\right)$, which we understand as representing the spacetimes which are nomologically admissible under the theory. As well as providing us with the solutions the action also gives us a precise methodology for defining the fundamental symmetries of the theory in terms of the Lagrangian $\mathcal{L}_{E H}$ and the Noether symmetry condition:

$$
\delta \mathcal{L}_{E H}=\partial_{\mu}\left(\epsilon^{\mu} \mathcal{L}_{E H}\right)
$$

which is satisfied for any active variation of the gravitational field variable (i.e. the metric tensor) induced by the infinitesimal coordinate transformations $x^{\mu} \rightarrow$ $x^{\mu}-\epsilon^{\mu}(x)$. The set of all such infinitesimal coordinate transformations forms the group of diffeomorphism of the manifold $\mathcal{M}$ that we will take to constitute 
the fundamental local symmetry group of the covariant formalism. ${ }^{2}$ Each of these two basic elements to the theory (i.e. solutions and symmetry group) are four dimensional and are understood as corresponding to four dimensional concepts; spacetimes and symmetries of spacetime. As such, the analysis of either is unlikely to elucidate much with regard to the specific role of time within the theory. Rather, we are better placed to understand the temporal structure of general relativity by passing from the covariant formulation to one which is predicate upon space and time rather than spacetime. We shall achieve this by focusing on the canonical formulation of general relativity.

The canonical formulation of general relativity has its origin in the work of Bergmann (1961) and Dirac $(1958,1964)$ towards the construction of a quantum theory of gravity and will be concisely presented according to the formulation of Arnowitt, Deser, and Misner (1962). We first make the assumption that the manifold $\mathcal{M}$ has a topology which is such that $\mathcal{M} \cong \mathbb{R} \times \sigma$ where $\sigma$ is a three dimensional manifold with arbitrary topology that we will again assume to be spatially compact and without boundary. ${ }^{3}$ What philosophical significance, if any, we should attach to this non-trivial topological requirement will be discussed in the following subsection. Next we define the foliation of $\mathcal{M}$ into hypersurfaces $\Sigma_{t}:=X_{t}(\sigma)$ where $t \in \mathbb{R}$ and $X_{t}: \sigma \rightarrow \mathcal{M}$ is an embedding defined by $X_{t}(x):=X(t, x)$ for the coordinates $x^{a}$ on $\sigma$. What we are interested in specifically is the foliation of a spacetime, $\mathcal{M}$, into spacelike hypersurfaces, $\Sigma_{t}$ - so we must restrict ourselves to arbitrary spacelike embeddings. The lengthly process of decomposing the Einstein-Hilbert action in terms of tensor fields defined upon the hypersurfaces and the coefficients used to parameterise the embedding (the lapse and shift below) then leads to a Lagrangian formulation of general relativity in terms of space and time rather than spacetime (see Thiemann (2007) for a full treatment). Finally, recasting this ' $3+1$ ' Lagrangian formalism into canonical terms gives us:

$$
S=\frac{1}{\kappa} \int_{\mathbb{R}} d t \int_{\sigma} d^{3} x\left\{\dot{q}_{a b} P^{a b}-\left[N^{a} H_{a}+|N| H\right]\right\}
$$

Here $q_{a b}$ is a metric tensor field on $\sigma$ and $P^{a b}$ its canonical momenta defined by the usual Legendre transformation. $N$ and $N^{a}$ are arbitrary multipliers called the lapse and shift. $H_{a}$ and $H$ are constraint functions of the form:

$$
\begin{aligned}
H_{a} & :=-2 q_{a c} D_{b} P^{b c} \\
H & :=\frac{s \kappa}{\sqrt{\operatorname{det}(q)}}\left[q_{a c} q_{b d}-\frac{1}{2} q_{a b} q_{c d}\right] P^{a b} P^{c d}-\sqrt{\operatorname{det}(q)} \frac{R}{\kappa}
\end{aligned}
$$

\footnotetext{
${ }^{2}$ As pointed out by Pons, Salisbury, and Sundermeyer (2010) general relativity actually admits the larger symmetry group of field-dependent infinitesimal co-ordinate transformations and so $\operatorname{Diff}(\mathcal{M})$ is properly a sub-group of the fundamental symmetry group. This difference will not be important for our purposes.

${ }^{3}$ See Lusanna (2011) and future work for discussion of the importance of this choice of boundary conditions.
} 
These are called the momentum and Hamiltonian constraints respectively and (like typical constraints) can be understood as defining a physical phase space $\Pi$ in terms of a sub-manifold (the constraint surface) within the extended phase space $\Gamma(q, P)$ :

$$
\Pi=\left\{\left(q_{a b}, P^{a b}\right)=x \in \Gamma \mid H_{a}(x)=0 ; H(x)=0\right\}
$$

Beyond their uncontroversial role in defining this sub-manifold, interpreting of the meaning of these constraint functions is a subtle business. In a typical constrained Hamiltonian theory (e.g. see Dirac (1964)) it is assumed that if, as in this case, the constraints are first class (i.e. have a vanishing Poisson bracket on the constraint surface with all the other constraints) then they should be taken to generate unphysical transformations of the canonical variables and to have their origin directly in the local symmetries of the covariant formalism. The extent to which canonical general relativity is not a typical constrained Hamiltonian theory in these senses and the consequent interpretation of the constraint functions is the decisive issue that will inform much of our discussion.

\subsection{Canonical vs. Covariant Formalisms}

To what extent does the the canonical formalism capture the same content as the covariant formulation? We can evaluated the answer to this question in two parts: i) is an equivalent set of solutions represented in the space and time formalism as was fixed by the spacetime formalism?; and ii) are an equivalent set of local symmetry transformations implemented upon the canonical phase space as where found to hold within the covariant configuration space (i.e. the space of four-metrics)?

Focusing on the first question first. Following Isham (1992) we have that given a Lorentzian spacetime as represented by the geometry $(\mathcal{M}, g)$ if this constraint equations (4) and (5) are satisfied on every spacelike hypersurface then $g$ will also satisfy the Einstein field equations. Conversely, we can also show that given a $(\mathcal{M}, g)$ that satisfied the Einstein field equations then the constraint equations will be satisfied on all spacelike hypersurfaces of $\mathcal{M}$. This means that the solutions presented to us by the two formalism are equivalent provided the covariant spacetime can be expressed in terms of a sequence of space-like hypersurfaces. This requirement is equivalent to insisting that spacetimes in question are restricted to be globally hyperbolic (see Geroch (1970)) and is of course directly connected to the topological restriction $\mathcal{M} \cong \mathbb{R} \times \sigma$ which was made in setting up the canonical formalism.

At first sight this might seem to render the canonical formalism fundamentally inadequate for describing the spacetime ontology as the covariant formalism. However, this objection that the requirement of globally hyperbolicity renders the canonical formulation of general relativity representatively deficient in comparison to the covariant formalism should not be overstated. By insisting that our spacetime is globally hyperbolic we are only requiring the existence of a Cauchy surface in $\mathcal{M}$, meaning that the only solutions that have been excluded are those inconsistent with the kind of basic notion of causality and determinism 
that we would prima facie have expected to hold within a classical theory anyway. The physical content of non-globally hyperbolic solutions seems hard to countenance since they included strange objects such as closed time-like curves and in terms of the empirical content of the theory nothing has been lost since all observational data refers to globally hyperbolic solutions. ${ }^{4}$ Within quantum gravity there is of course the possibility that may need access to different kinds of topologies - or, in fact, perhaps even topology changes! However, the reasoning behind lifting the topological requirement at a quantum level do not impinge on its classical status. To the extent to which the solutions of covariant general relativity can be understood as representing physically reasonable spacetimes the solutions of covariant general relativity can equally be thought of representing these spacetimes (albeit in terms of space and time).

Still, it $i s$ true that since canonical general relativity is well defined on a set of solutions which is a subset of those of covariant general relativity in moving from one formalism from the other we are removing from our theoretical toolkit the ability to represent a class of nomologically possible worlds. Furthermore, although these worlds might seem unreasonable because of their strange causal structure, to exclude them in principle from a philosophical analysis of the nature of time in general relativity would be seriously begging the question. Asserting a causal censorship condition excluding the non-globally hyperbolic solutions as an additional law of nature is a highly non-trivial move which we will not here propose to make. Rather, one straight forward option is to invert the supposed deficiency into a strength and make global hyperbolicity a prediction rather than a restriction. Such a move depends on our ability to see the canonical formalism on an equal footing to its covariant counterpart, and not as purely parasitic upon it. This we can do by pointing to the fact that as shown by Hojman, Kuchar, and Teitelboim (1976) - it is possible to derive canonical general relativity without passing through the covariant formalism. Such a manoeuvre will be crucial to the assessment of our second denial of time and we will be further discussed in $§ 4.3$. Alternatively, we can simply fall back on a weakened stance: this analysis and its conclusions with regard to the nature of time carry with them a global parenthesis of given the restriction to the globally hyperbolic sub-set of solutions. Since our principal object is to examine issues relating to diffeomorphism symmetry and the ontological status of a linear one dimensional notion of temporality our discussion can bear this qualification without any undue burden or inconsistency.

More significant to our purpose is the relationship between the respective local symmetry transformations of the two formalisms. Whereas, as discussed above, the covariant action is invariant under the full set of spacetime diffeomorphisms $\operatorname{Diff}(\mathcal{M})$, in the canonical formulation it is only the sub-set of these which is realised. This sub-set can be shown (e.g. Pons et al. (2010)) to be

\footnotetext{
${ }^{4}$ For an analysis of the connection between non-globally hyperbolic spacetimes, closed time-like curves and the possibility of time machines see Smeenk and Wuthrich (2009)
} 
infinitesimal coordinate transformations $x^{\mu} \rightarrow x^{\mu}-\epsilon^{\mu}(x)$ such that:

$$
\epsilon^{\mu}(x)=n^{\mu}(x) \xi^{0}+\delta_{a}^{\mu} \xi^{a}
$$

where $n^{\mu}=\left(N^{-1},-N^{-1} N^{a}\right)$ and here the $\xi^{\mu}$ are taken to be arbitrary functions of the coordinates. From the persecutive of the derivation of canonical from covariant general relativity the basis of this discrepancy between the symmetry transformations realised in the two formalism is well understood - it can be explained in terms of the spacelike nature of the otherwise arbitrary embedding (see Isham and Kuchař (1985)) or (relatedly) in the context of noncomplete projectability between the symmetry transformations defined in the relevant tangent bundle and cotangent bundle structures (see Pons, Salisbury, and Shepley (1997)).

Alternatively, we can consider the elegant and important derivation of these canonical symmetry transformations purely in terms of a deformation algebra pertaining to spacelike hypersurfaces embedded in a Riemannian spacetime (Teitelboim (1973)). This treatment gives us a basis for the canonical symmetry transformations independent of the covariant theory and implies that we can understand them as encoding physical content not purely parasitic upon four dimensional diffeomorphism symmetry. Crucially, this treatment also makes clear the deep connection between the form of the constraints and the nature of the symmetries. In fact, what is shown is that this canonical symmetry group (known as the Bergman-Komar group $\mathcal{B K}$ ) is and must be generated by constraints of the specific form (4-5) which will inevitably satisfy the constraint algebra:

$$
\begin{aligned}
\left\{\vec{H}(\vec{N}), \vec{H}\left(\vec{N}^{\prime}\right)\right\} & =-\kappa \vec{H}\left(\mathfrak{L}_{N_{a}} N_{a}^{\prime}\right) \\
\{\vec{H}(\vec{N}), H(N)\} & =-\kappa H\left(\mathfrak{L}_{N_{a}} N\right) \\
\left\{H(N), H\left(N^{\prime}\right)\right\} & =s \kappa \vec{H}\left(F\left(N, N^{\prime}, q\right)\right)
\end{aligned}
$$

where $H(N)$ and $\vec{H}(\vec{N})$ are smeared versions of the constraints (e.g. $\vec{H}(\vec{N}):=$ $\left.\int_{\sigma} d^{3} x N^{a} H_{a}\right)$ and $F\left(N, N^{\prime}, q\right)=q^{a b}\left(N N_{b}^{\prime}-N^{\prime} N_{, b}\right)$. The presence of structure functions on the right hand side of $(10)$ means that strictly $\mathcal{B K}$ is not a group (and the constraint algebra is not technically an algebra) and of course emphasises that $\operatorname{Diff}(\mathcal{M}) \neq \mathcal{B K}$.

Despite these important differences the symmetries of the covariant and canonical formalism can in fact be shown to be physically equivalent since given a solution to the equations of motion within the canonical formalism the action of $\mathcal{B K}$ will coincide with that of $\operatorname{Dif} f(\mathcal{M})$ (for diffeomorphisms connected with the identity). Thus, at a classical level at least there is no detectable difference. We can in fact explicitly construct a canonical symmetry generator acting upon the space of solutions that maps between the same diffeomorphically related spacetimes that we take to be symmetry related in the covariant formalism (see Pons et al. (2010) and references therein for more details). Thus, so long as we are concerned with globally hyperbolic solutions, the two formalisms admit identical symmetry relations. 


\subsection{Time and the Riddle of the Hamiltonian Constraints}

In the discussion above the constraints of the canonical formalism were found to be involved in fixing both the dynamics and symmetries to be in accordance with the physics of covariant general relativity. This dual symmetry/dynamics aspect leads to much confusion and complexity with regard to the constraints - in particular the Hamiltonian constraint - and is at the root of the problem of time in canonical gravity. Whereas the momentum constraints can be understood unambiguously as implementing infinitesimal diffeomorphisms on phase space, the role of the Hamiltonian constraints in this context is far more opaque. We can see this explicitly by considering the form of the Poisson brackets between each constraint and the canonical variables. For the momentum constraints it takes the form:

$$
\begin{array}{r}
\left\{\vec{H}(\vec{N}), q_{a b}\right\}=\kappa\left(\mathfrak{L}_{\vec{N}} q_{a b}\right) \\
\left\{\vec{H}(\vec{N}), P^{a b}\right\}=\kappa\left(\mathfrak{L}_{\vec{N}} P^{a b}\right)
\end{array}
$$

The appearance of the Lie derivative on the right hand side of each equation indicates that these constraints can be understood as purely generating infinitesimal diffeomorphisms of the phase space variables. In fact it means that these constraints can on their own be understood as implementing the Lie group of diffeomorphisms of the space-like hypersurface $\sigma$ (Isham and Kuchař (1985)).

The Hamiltonian constraints in, stark contrast, have a phase space action which seems, prima facie, manifestly dynamical. For any specification of the lapse they affect an infinitesimal phase space transformation from the canonical variables characterising a given three geometry to those describing a second three geometry which is dynamically subsequent. More careful analysis however reveals a dual role within which the seeds of our conceptual enigma are sown. We can consider its explicit action upon an embedded canonical momenta variable. Such a variable is so called because it is the canonical conjugate of an metric variable $q^{\mu \nu}$ which is a tensor field (the first fundamental form) defined on the embedded hypersurface $\Sigma_{t}$. This new metric variable can be expressed purely in terms of spatial vector fields on $\Sigma_{t}$ and the usual metric variable on $\sigma, q^{a b}$ (see Thiemann (2007, Eq. 1.1.16)). The new momenta variable can be written in terms of $q^{\mu \nu}$ together with it and another spatial tensor field on $\Sigma_{t}$ (the second fundamental form). An elegant calculation by Thiemann (2007, pp.54-6) yields the explicit expression:

$$
\left\{H(N), P^{\mu \nu}\right\}=\frac{q^{\mu \nu} N H}{2}-N \sqrt{\|q\|}\left[q^{\mu \rho} q^{\nu \sigma}-q^{\mu \nu} q^{\rho \sigma}\right] R_{\rho \sigma}^{D+1}+\mathfrak{L}_{N n} p^{\mu \nu}(13)
$$

with $R_{\mu \nu}^{4}$ the Ricci 4-tensor. The first term on the right is zero on the physical phase space (defined by satisfaction of the constraints) and is therefore unimportant. The second is zero for solutions to the equations of motion and thus we have that on shell the Hamiltonian performs the role of generating infinitesimal diffeomorphisms. Whereas the diffeomorphisms associated with the momentum constraints can be understood as purely kinematical symmetries of 
the three geometries $\sigma$ (irrespective of whether the equations of motion hold) those associated with the Hamiltonian constraint are symmetries of not just entire spacetimes but of spacetimes which are solutions. For a given solution and an embedded hypersurface the constraint generates a local deformation of the hypersurface. Collectively such an action is equivalent to the refoliation of a spacetime and therefore to the generation of a different unphysical splitting of spacetime into space and time. However, the solutions themselves are consequences of the dynamical role that the Hamiltonian constraint plays in the context of three geometries considered on their own rather than as embedded in a spacetime. Thus, to maintain both the fundamental symmetry of the theory and the dynamics we must appreciate the dual, context dependent role of the Hamiltonian constraints. ${ }^{5}$ In the remaining discussion we will explore the narrow path that traverses failure to appreciate either side of this duality and, after observing the perils of falling into the abyss below, will come upon a fork that forces us to choose between retaining a weaker Machian notion of time at the cost of global scale and dispensing with time altogether.

\section{Denial I: Reductive Temporal Relationalism}

\subsection{Gauge Theory and Symplectic Reduction}

Motivation for a particularly influential (but ultimately unpersuasive) argument towards the denial of time in canonical general relativity derives from the consideration of the otiose representative structure constituted by the Hamiltonian formulation of a generic gauge theory. In order to frame this argument adequately we will briefly consider the structure of these theories in general without any particular reference to the Hamiltonian constraints of canonical general relativity and the important subtleties that go along with them.

Consider a constrained Hamiltonian theory constituted by a phase space $\Gamma$ parameterised by $n$ canonical coordinates $(p, q)$, a Hamiltonian functional $H(p, q)$ and a set of $m$ constraint functions $\varphi_{i}(p, q)=0$. It is well know that such a theory may be taken to corresponds to a physical system with gauge freedom - i.e. those which have a Lagrangian formulation in which the action is invariant under some local symmetry group, form example Electromagnetism or a YangMills theory. ${ }^{6}$ Geometrically we can characterise such a generic constrained

\footnotetext{
${ }^{5}$ It is important to note that this key aspect to our analysis represents a departure from both the received and dissenting view on this matter (although it is close to the spirit of Pons et al. (2010)). Whereas, the received view is that the Hamiltonian constraints purely generate unphysical transformations (e.g. Rovelli (2004)), the dissenting view (notably defined in Barbour and Foster (2008) but also associated with Kuchař $(1991,1992)$ ) is that the constraints' action is purely physical in character. The latter view is supported by the argument (which I do not personally find entirely convincing) that Hamiltonian constraints should not be understood as being involved in re-foliating a spacetime since neither the spacetime nor the foliation are there before they act (Julian Barbour, private correspondence, August 2011).

${ }^{6}$ See Dirac (1964) or Henneaux and Teitelboim (1992). We presume here that the constraint featured are primary, first class constraints whose action can be unambiguously connected to the cotangent bundle projection of a Lagrangian gauge group defined as acting on the relevant
} 
Hamiltonian theory in precise terms. Firstly, we have by definition that the phase space $\Gamma$ is equipped with a Poincaré one form $\theta$ and that the exterior derivative of this one form gives us a symplectic (i.e. closed and non-degenerate) two form $\omega{ }^{7}$ This allows us to defined the symplectic geometry $(\Gamma, \omega)$. We can then define, by satisfaction of the constraint conditions, the $n-m$ dimensional sub-manifold $\Pi=\left\{(p, q) \in \Gamma \mid \forall_{i}: \varphi_{i}(p, q)=0\right\}$. The sub-manifold, which we designate the physical phase space since its complement contains unphysical states which are in violation of the symmetries of the theory, has a geometry endowed by the exterior derivative of the restriction of $\theta$ to $\Pi$. In the cases in which we are interested it is found that that gives rise to a presymplectic (closed and degenerate) form $\Omega$ and therefore a presymplectic geometry $(\Pi, \Omega)$. Such geometries are distinguished by their degenerate structure - with the integral curves of the vector fields that make up the null vector space (or Kernel) of $\Omega$ partitioning $\Pi$ into a set of transverse sub-manifolds called gauge orbits. In physical terms each of these orbits have the significant feature that the all of the constituent points are physically indistinguishable - they correspond to an identical value of the Hamiltonian functional. Furthermore, paths in the physical phase space which differ only as to a transformation along a gauge orbit will necessarily correspond to identical values of the canonical action and will therefore also be indistinguishable.

Given that we make the usual interpretation of points in the (physical) phase space as representing distinct instantaneous physical states the above feature is a form of indeterminism (or underdetermination) since given an initial specification of physical states the formalism does not fix a unique continuation. This would seem unsatisfactory for the case of classical theories, such as electromagnetism, where the relevant measurable quantities are manifestly deterministic. The natural response to such circumstances is to point to inadequacy within our representative formalism rather than the characterisation of the connection between what is real and what is measurable within our theories. We assert that there is 'surplus structure' within our formalism as embodied precisely by the directions defined by the gauge orbits. The most obvious methodology for controlling this excess is to classify these directions as unphysical and use points in the space of gauge orbits to give us a unique representation of physical states. ${ }^{8}$ Formally we may construct this space of gauge orbits or reduced phase space in terms of the quotient manifold that results from the application of a symplectic reduction procedure to the physical phase space П. For simple constrained Hamiltonian theories this reduction enacted simple by taking the

tangent bundle. As discussed above this is of course explicitly not the case for canonical general relativity

${ }^{7}$ For a powerful yet concise introduction to these ideas Arnold, Kozlov, and Neishtadt (1988) is highly recommended

${ }^{8}$ Less obviously we might instead weaken the representative relationship between points and states via the introduction of some notion of anti-haecceitism. This strategy will be examined carefully within the particular context of time in canonical general relativity as discussed in $\S 4.2$ 
quotient $\Pi$ by the kernel of $\Omega$ - see Gotay, Nester, and Hinds (1978) for the more complex case. Either way, it can be proved (Souriau (1997)) that the space of gauge orbits that results by the application of symplectic reduction to the physical phase space has a symplectic geometry $\left(\Gamma_{R}, \omega_{R}\right)$ and inherits a Hamiltonian functional from the physical phase space. It is therefore equipped to describe dynamics and since we have removed the null directions and since the space has a non-degenerate structure it is of course not afflicted with the kind of indeterminism mentioned above. If we assign to points in the reduced phase space the role of representing unique physical states then the formalism is now such that any initial specification will also imply the provision of a unique continuation.

By passing to the reduced phase space of a constrained Hamiltonian theory we reap the reward of a formalism trimmed of any superfluous representative structure. This has led some authors to argue that we should endow the reduced space with a privileged status. In particular Gordon Belot and John Earman (Belot (1999, 2000, 2003, 2007); Earman (2002, 2003); and Belot and Earman $(1999,2001))$ have argued that we should consider the reduced phase space as the fundamental dynamical arena of a gauge theory. As applied to a generic gauge theory this form of reductionism, although open to a number of philosophical objections (e.g. see Rickles (2007)), is a viable option and is to a large degree supported by the various techniques of canonical quantisation for gauge theories - all of which can be seen to be predicated upon the reduced phase space. ${ }^{9}$ However, for the specific case of the Hamiltonian constraints which feature in canonical general relativity (as well as simple theories which are reparameterisation invariant Thébault (2011b)) such reductionism rests on an inappropriate interpretation of the Hamiltonian constraint as a pure gauge generator. We will examine this crucial issue more carefully after first giving a second motivation for symplectic reduction that is specific to canonical general relativity and is based on a form relationalism as to the spacetime concepts found within the theory.

\subsection{Reductive Spacetime Relationalism}

The philosophical doctrine of relationalism with regard to space and time has it roots in the early modern natural philosophy of Descartes, Leibniz and Huygens but (arguably) takes its most precise form in the work of Mach and Poincaé (Barbour (2009)). In essence, it is a position as to the relative ontological status of relations between material bodies and the entities or objects constituted by space and time themselves. A relationalist is taken to hold that the relations are primary and that space and time are merely derived or abstracted based upon them. In the context of a theory containing a concept of dynamical spacetime such as general relativity it is not entirely clear what relationalism as it was originally conceived should be taken to mean and the modern

\footnotetext{
${ }^{9}$ See Belot $(2007, \S 4.3)$ and Thébault (2011a) for discussion of the relationship between quantisation and reduction
} 
philosophical discussion is replete with positions which are taken to be either pro- or anti- some version of relationalism. Our purpose here will not be to survey this literature nor explicitly analyse its connection with the indeterminism issue of the previous section in terms of the famous hole argument. ${ }^{10}$ Rather, we will concern ourselves the notion of reductive spacetime relationalism that is presented by the relevant authors in their argument towards our first denial of time. A second, importantly different notion of temporal relationalism will be discussed in $\S 4.2$.

Among others, Belot and Earman $(1999,2001)$ hold that the essance of spacetime relationalism within general relativity should be taken to be the denial of a fundamental ontological role for spacetime points. Such points are of course represented within a covariant formalism by the coordinatisation of the manifold $\mathcal{M}$ and will therefore be given distinct representations within different coordinatisations. If we assume that cross identification between points within qualitatively identical spacetime models - i.e. with the same geometry - can be taken to ground a real difference between these models (i.e. they may differ solely haecceitistically), then relationalism can be understood in terms of the denial of exactly such difference on the grounds that spacetime points do not a fundamental ontological status. ${ }^{11}$ A spacetime relationalist is thus someone who will 'deny that there could be two possible worlds with the same geometry which differ only in virtue of the way that is geometry is shared out over existent spacetime points' Belot and Earman (2001, p.18). In the context of the covariant formalism this means that two geometries $\left(M, g_{\mu \nu}\right)$ and $\left(M^{\prime}, g_{\mu \nu}^{\prime}\right)$ which solve the Einstein field equations and are related by an element of $\operatorname{Diff}(\mathcal{M})$ are considered the same physically possible situation. This is because the difference between them is exactly in terms of the coordinatisations rather than the geometrical structure; and therefore the ontologies which they are taken to represent can differ (if they differ at all) only with respect to the role played by the spacetime points. By endorsing such a Leibniz equivalence type principle, Belot and Earman disavow this difference ${ }^{12}$. Furthermore, by cutting down the class of distinct possibilities to include only geometries which are members of different diffeomorphically related equivalence classes we have implicitly performed a reduction with respect to our fundamental representative space. Rather than considering the space of Riemannian four-geometries corresponding to four-metrics which solve the Einstein field equations as our basic arena for representing the world we instead should consider the quotient of that space by the group of four dimensional diffeomorphisms. Thus, we can see Belot and Earman's arguments as leading us from relationalism to reduction: they are reductive relationalists.

What does this reductive form of spacetime relationalism mean in the con-

\footnotetext{
${ }^{10}$ See Rickles (2007) on both counts

${ }^{11}$ We will, for the time being, defer the discussion of anti-haecceitist variants of relationalism. See $\S 4.2$

${ }^{12}$ There are, of course, other ways of formulating such a principle that do not have the same implications for possibility reduction. For instance that suggested by Saunders (2003)
} 
text of the canonical formalism? If we focus our attention on the role of spatial points then we have a clear answer. In analogy to the spactime case spatial points are represented in terms of the coordinatisation of a manifold, in this case the three dimensional manifold $\sigma$. Furthermore, the action of the theory is invariant under the group of three dimensional diffeomorphisms of this manifold, $\operatorname{Diff}(\sigma)$, and so a reconstruction of the argument above can be made for this case. Explicitly, since we have that two canonical solutions which differ solely on the basis of the application of an element of $\operatorname{Diff}(\sigma)$ to one of their constituent three geometries are physically identical then asserting the existence of spatial points will violate a Leibnizian type principle of equivalence of exactly the same type as that in introduced via the quote from Belot and Earman (2001)above. Thus, a reductive relationalist with regard to space will endorse a reduced space of three geometries as representatively fundamental within the canonical formalism. Since we have from above that $\operatorname{Diff}(\sigma)$ is implemented on phase space by the action of the momentum constraints we know that precisely the reduced space we are looking for can be achieved by quotienting out the gauge orbits associated with those constraints according to the symplectic reduction procedure above. This, in fact, leads us directly to Wheeler's superspace (see Wheeler (1968), Giulini (2009)) upon which a formulation of canonical general relativity would be constituted according to this brand of spatial reductive relationalism.

With regard to time things are, as ever, far more complicated. One might hope to translate a position of spacetime relationalism as expressed in terms of the covariant formalism into a position of spatial relationalism plus temporal relationalism as expressed in terms of the canonical formalism. Building on the ideas of the previous paragraph we would hope to disavow the fundamental status of temporal points by enforcing ontological equivalence between solutions which differ only as to the way in which the four dimensional geometrical structure is 'shared out' over these points. Thus in effect performing a reduction of paths in superspace such that those which differ only as to how time is labelled are classified as the same path and a new double reduced representative space is arrived at. Unfortunately such a naive implementation of reductive temporal relationalism is neither possible nor adequate to our purpose.

Although we have assumed that the spacetime manifold $\mathcal{M}$ has a topology which is such that $\mathcal{M} \cong \mathbb{R} \times \sigma$ and therefore that the temporal dimension is represented in terms of the real line, the complication of foliation invariance means that the arbitrariness with regard to time is not fully captured merely by global temporal relabelling - i.e. by the one dimensional diffeomorphisms group $\operatorname{Diff}(\mathbb{R})$. Furthermore, unlike spatial diffeomorphisms these 'temporal diffeomorphisms' have no representation at the level of a constraint function acting of phase space points or for that matter even phase space paths and so (in the conventional formalism) is impossible to frame this naive temporal relationalism simply in terms of a reduction procedure.

Foliation invariance means that the theory is invariant under the set of local temporal relabelling of each point on each space-like hypersurface of which the global temporal relabellings discussed above form a sub-set. To be consistent with the notion of reductive spacetime relationalism defined above it is the tem- 
poral points that constitute this local labelling that must be excluded from our ontology via a Leibniz equivalence inspired quotienting operation. In the case of local temporal relabelling (unlike the global case) we do have a canonical constraint function that can be associated with the relevant symmetry, the Hamiltonian constraint. However, as discussed above the connection between these constraints and refoliation symmetries can only be made precise at the level of paths in the physical phase space which are also solutions to the equations of motion. In the context of its action on phase space the Hamiltonian constraint generates evolution. Thus, although it might seem at first sight that reducing out the action of the Hamiltonian constraint (on superspace) will achieve the object of reductive spacetime relationalism within the canonical formalism, our understanding of the constraints dual role leads to immediate scepticism on this count. The object of reductive relationalism with regard to time in particular, is to construct a representative arena in which the distinct possibilities entailed by the existence of temporal points have been removed. Within the context of canonical general relativity such a reduction makes sense (at least in principle) at the level of entire histories related by a refoliation symmetry. However, it is difficult to seem how it can possibly be achieved by a reduction of phase space since such symmetries can not be represented in terms of the relationship between points on this space. It is exactly this kind of phase space reduction with regard to the Hamiltonian constraint which Belot and Earman argue implements their reductive spacetime relationalism within the canonical formalism, and to which we now turn.

\subsection{Dynamical Trivialisation and the Isomorphism Argument}

We thus have two distinct but connected motivations for enacting a symplectic reduction of the phase space of canonical general relativity with regard to the Hamiltonian constraints. Firstly, we have the argument from indeterminism and surplus structure - it is assumed that as for the case of other theories with first class constraint, the sub-manifolds defined by the integral curves of the null vector fields associated with the Hamiltonian constraints will form gauge equivalence classes. Thus, as for the generic case, the unreduced formalism will poses an excess representation of physical states such that an initial specification of phase space points will admit multiple physically identical but mathematically distinct continuations. By reducing out the action of these constraints we will remove both this indeterminism and the redundant representative structure that enables it. Secondly, we have the motivation from reductive spacetime relationalism - we wish to reduce our possibility space such that differences entailed by distinct coordinatisations of the same fundamental geometrical structure are no longer encoded. Specially, in addition to removing the representation of spatial points, via reduction with respect to the momentum constraints, we also want to remove the local temporal labellings that play the role of representing temporal points within the theory. To an extent the reduction that Belot and Earman have in mind does achieve both these purposes, however, it comes at a dire cost to the representative adequacy of our formalism such that we are only equipped to represent dynamically trivial universes. 
As detailed in $\S 2.3$ and mentioned above the role of the Hamiltonian constraints within canonical general relativity is essentially a dual one. When considered as acting on purely on a three geometry (as represented by a phase space point) they generate dynamical evolution and when considered as acting on space-like hypersurface embedded within a solution they generates infinitesimal diffeomorphisms. The latter role means that the constraint can be considered responsible for generating refoliation symmetries and allows us to understand how the four dimensional diffeomorphism symmetry is (to a certain extent) implemented canonically. However, the former role cannot be discounted since without it the solutions within which the hypersurfaces are embedded cannot be defined. Moreover, the gauge orbits associated with the constraints action on phase space are in fact closer in character to solutions themselves and are explicitly not equivalence classes of solutions since a point with the orbit is associated with a three not four dimensional object. Still by classifying all phase space points within these sub-manifolds as representing the same state we will ensure that any pair of three geometries which are contained within solutions related by a refoliation symmetry will be classified as equivalent. Thus symplectic reduction will remove the indeterminism related to that symmetry. It will, of course, also therefore mean that the reductive temporal relationalist desire to pass to a representative space that excludes distinct local temporal labellings will also have been achieved.

In addition to these two primary goals, however, this reduction has the unintended consequence that all dynamically related three geometries are classified as representing the same state. This is because the orbit that is quotiented is, as it must be by the nature of the Hamiltonian constraints phase space action, composed of every state that can be accessed via the 'many fingered' time evolution the the theory allows for in terms of the action of the Hamiltonian and the arbitrariness of the lapse function. By reducing the representative capacity of the orbit down to a single state we pass from many fingers to no fingers - and not one finger! Furthermore, since we have not respected the dynamical role of the Hamiltonian in a phase space context by passing to the reduced space we will have classified states which are physically distinct members of a given solution as identical. This is exactly to treat the current state of the universe and its state just after the big bang as identical (contra the claims of Belot (2007)).

We can illustrate the dynamical triviality of the reduced space explicitly by considering the presymplectic structure of the physical phase space of canonical general relativity. ${ }^{13}$ The phase space $\Gamma$ is a Poisson manifold and it is therefore equipped with a canonical (four) form $\tilde{\theta}$. Together all the constraints of the theory (i.e. the Hamiltonian and momentum constrains together) define the physical phase space, $\Pi$, and the restriction of our original form to this surface will induce a second form $\theta=\left.\tilde{\theta}\right|_{\Pi}$. The exterior derivative of this gives us a (five)

\footnotetext{
${ }^{13}$ We here follow (Rovelli, 2004, §4.3). See his treatment for more details and the appropriate references
} 
form $\omega=d \theta$ and since $\omega$ has null directions it is presymplectic and therefore defines a presymplectic geometry $(\Pi, \omega)$. If we define the orbits of $\omega$ to be four dimensional surfaces $\bar{\gamma}$ in $\Pi$ such that the quadritangent $X$ to the orbit is in the kernel of $\omega$ (i.e. $\omega(X)=0$ ) then we can identify the $\bar{\gamma}$ with solutions of the Einstein field equations. Thus since it is exactly these null directions that are removed in any quotienting operation a total reduction of the theory will involve the reducing out of the solutions. Since we know from above that the role of the momentum constraints can be understood in conventional gauge theoretic terms, it is clear that it is the Hamiltonian constraints that so entangle the dynamics with the gauge orbits. Reducing out their action will lead to a dynamical trivialisation of the theory.

A single line of argument is available in defence of total constraint reduction in canonical general relativity against the charge of dynamical trivialisation. Belot (2007) argues that rather than seeing the reduced phase space as dynamical trivial in sense outlined above we should instead reinterpret it as a space of diffeomorphically invariant histories. Thus, we would enable both reductive temporal relationalism and the avoidance of indeterminism but without the cost of trivialising our dynamics. Belot's argument relies on the existence of a canonical isomorphism between the fully reduced phase space and a space of diffeomorphically invariant spacetimes defined via the covariant formalism and we shall therefore dub it the isomorphism argument.

Essentially it runs as follows. Consider a system which does not display any gauge freedom. Its dynamics can be described in terms of a space of solutions to the Euler-Lagrange equations $\mathcal{S}$ or in terms of set of curves in a phase space $\mathcal{I}$ with the usual symplectic structure. Although there exists an isomorphism between points in these two spaces they have distinct representational roles - a point in $\mathcal{S}$ represents an entire history of our system and a point in $\mathcal{I}$ represents an instantaneous state of the system. According to Belot this distinction is grounded in terms of the existence of a distinct isomorphism between any instant in a slicing of a solution $\gamma \in \mathcal{S}$ into a sequence of Cauchy surfaces $\Sigma_{t}$ and the point in the space of phase space which contains the instantaneous data relevant to that value of the temporal parameter. Clearly, under this interpretation if the system is taken to be the whole universe then points in $\mathcal{I}$ should be considered as representing distinct instantaneous states of the world and those in $\mathcal{S}$ should be considered as representing worlds composed out of such states. Now, for a standard gauge theory of the type discussed in $\S 3.1$ such an interpretation can no longer be justified in these terms. Rather than having a one-to-one map which confers representative equivalence between each time slice of solution and a point in phase space, for each slice of a given solution we have a one-to-many map with its target an entire gauge equivalence class of points in phase space. However, if we pass to a reduced phase space $\mathcal{I}_{R}$ via symplectic reduction as well as constructing a reduced solution space $\mathcal{S}_{R}$ via an analogous reduction process (i.e. quotienting out the action of the Lagrangian gauge group) then we recover our distinct isomorphism per time slice and therefore also our argument towards the different representational roles of the two spaces - the former for instants the latter for entire worlds. The case of general relativity - as an atypical gauge 
theory - is crucially different. Because of the nature of the diffeomorphism group points in the reduced space of solutions cannot be individually decomposed into slices and this means that only a single isomorphism exists between each of these diffeomorphically invariant solutions and points in the reduced phase space. This, Belot argues, means that we should interpret them as representationally equivalent spaces. Thus, the reduced space is dynamically non-trivial since it can be taken to represent universes which contain evolution.

Although, innovative and to some extent insightful the isomorphism argument of Belot is problematic in a number of respects. Firstly, it seems to rest on the non-sequitur that since the existence of a distinct isomorphism per time slice gives us grounds to fix distinct representational roles for $\mathcal{I}_{R}$ and $\mathcal{S}_{R}$, the non-existence of such a family of isomorphisms implies that the two space are should be taken to be representatively equivalent. Completely besides the nature of the mappings that exist between them we have very good reasons for asserting that solutions represent worlds and phase space points represent instants - the variational basis upon which the two structures are defined and the different form of the relevant boundary conditions to name just two. Just because in the case of general relativity we no longer have access to one argument towards their representational inequivalence does not indicate that we no longer have any arguments available at all! Furthermore, the existence of an single isomorphism between points in two representative spaces is far from a sufficiency condition for them playing equivalent roles (although it could in some cases be taken to be necessary) since we can trivially find such relationships between manifestly inequivalent structures - two books with the same number of words for example. It makes far more sense for the representational role of a space within a theory to be fixed primarily by its relationship to the representative structures from which it is derived rather than a space utilised in the context of a different formalism. For the case of general relativity, therefore, it is far more appropriate to consider the relationship between the reduced phase space and the unreduced phase space as fixing the formers representational role. If we accept Belot's interpretation of the reduced space as a space of histories then we seem to be forced into asserting that points in the unreduced space are also representative of four dimensional histories and this is manifestly inconsistent with the ADM procedure that lead to the construction of this space. Rather, since we know by definition that a point in the unreduced phase space corresponds to a three geometry we should take points in the unreduced space to represent instantaneous states and curves in this space to represent entire four dimensional histories. By passing to a quotient of this space we are classifying sets of points as equivalent and so representatively speaking we are classifying groups of instantaneous states as equivalent. To be consistent with both the representative role of the space from which it is constructed and the manner of its construction the reduced phase space must be interpreted as representing instantaneous states and our charge of dynamical triviality against reductive temporal relationalism cannot be avoided.

We therefore see that reductive temporal relationalism and the form of denial of time which it implies is an at best problematic and at worst fatally flawed 
position. It leads to an interpretation of the formalism of canonical general relativity which is not adequate as a representative framework for describing the world since it admits only static universes. The crucial question is now which aspects of the interpretation are responsible for driving us into such a conceptual cul-de-sac? Was it the temporal relationalism or the reductionism which was the cause of the problem? In the next section we will investigate a different conception of temporal relationalism, in part, with the object of settling this matter.

\section{Denial II: Machian temporal relationalism}

\subsection{Machian dynamics and the Hamiltonian constraints}

A second and quite different perspective on time within general relativity is enabled by the Machian temporal relationalism of Barbour (1995, 1994, 2009). The principal element of this form of relationalism with regard to time is not an objection to temporal points forming part of our basic ontology, nor even the assertion of a Leibnizian equivalence principle such that any universes related by temporal symmetries must be judged to constitute distinct possibilities although consistency with these other relationalist dictates is implicit. Rather for Barbour the fundamental edict of temporal relationalism is that time should be 'an abstraction, at which we arrive by means of the changes of things; made because we are not restricted to any one definite measure, all being interconnected' (Mach (1960)). This Machian viewpoint on time can be seen as an imperative to try to construct (or at least restructure) our theories in such a way that time does not appear within the basic structure of the theory but is a well defined notion at a derived or emergent concept level. Thus, as well as a position as to what time is not, the Machian variant of relationalism is a position as to what time is. Particularly, important to both Barbour's interpretation of Mach (which he shares with Mittelstaedt (1976) and his own philosophy, is that the relational definition of time is a holistic and democratic one based upon contributions from all the motions within the universe. We will take this to mean that a theory or interpretation of a theory that is temporally relational in a Machian sense should provide us with a distinct definition of time for any dynamical history of the universe.

Although it clearly starts from a different perspective, there is a degree of coherence between this form of temporal relationalism and that predicated upon the denial of temporal points discussed above. There is no room within the ontology of a Machian theory for any basic temporal structure since this structure must itself be abstracted out of the ontology and not form part of it. Furthermore, it also seems safe to presume that the democratic nature of the process by which time is abstracted will be such that universes related by temporal symmetries must bear the same emergent notion of temporality. We can therefore expect that even at the level of an abstracted concept of time a Leibnizian equivalence principle should be satisfied.

So much for what it means to be a Machian temporal relationalist in principle. In practice, we have already seen a formal basis sufficient to establish such 
a position can easily be achieved within non-relativistic mechanics by switching to the formalism of Jacobi as formulated above. Again, restating the key ideas for the convenience of the reader. The Jacobi Hamiltonian takes the form

$$
H_{J}=\sum_{i} p_{i} \cdot q_{i}^{\prime}-L_{J}=N_{J} h_{J}
$$

where we define the Jacobi Hamiltonian constraint as

$$
h_{J}=\frac{1}{2} \sum_{i} p_{i} \cdot p_{i}+V-E=0
$$

and the lapse is an arbitrary function of of the time label $\tau$. The form of these equations is very suggestive of the canonical formulation of general relativity introduced above. We have a Hamiltonian constraint that is connected with arbitrariness in temporal labelling, and we a Hamiltonian that is made up only of a constraint and an arbitrary multiplier. What is particularly important for our purposes is how we should interpret the action of the Jacobi Hamiltonian constraint upon phase space. Explicitly we have that (provided the constraint is satisfied)

$$
N_{J}\left\{q_{i}, h_{J}\right\}=\frac{\delta q_{i}}{\delta \tau}
$$

which indicates that for any specification of the Jacobi lapse the Jacobi Hamiltonian will effect an infinitesimal phase space transformation from the canonical variables characterising a given instant in time to those describing a second instant that is dynamically subsequent.

This is in close analogy to the dynamical role of the Hamiltonian constraints of canonical general relativity. However, as in the relativistic case, this Poisson bracket also encodes a symmetry generating role in that, strictly speaking, the transformation that $h_{J}$ generates is unphysical because of the dependence on the arbitrary parameterisation encoded in the lapse. Thus again we have evolution enacted by a constraint and thus our dynamics and our temporal symmetry are entangled. In the case of Jacobi's principle, there is a straightforward methodology for disentangling them in the context of Machian temporal relationalism. As mentioned above, as well as the preclusion of external temporal parameters within our mechanical theory, the Machian temporal relationalist position involves a positive idea of time as an equitable measure that can be abstracted from dynamics. Jacobi's principle admits exactly this notion of temporality because we may naturally specify an emergent temporal increment:

$$
N \delta \tau=\sqrt{\frac{T}{(E-V)}} d \tau=\sqrt{\frac{\delta q_{i} \cdot \delta q_{i}}{2(E-V)}}
$$

In Lagrangian terms this notion of ephemeris time is introduced by choosing $\tau$ such that $T=E-V$. This then leads to the emergent temporal increment $\delta \tau$ and allows us to identify the Hamiltonian lapse with $\sqrt{\frac{T}{(E-V)}} \cdot 14$ The ephemeris

${ }^{14}$ Thanks to Julian Barbour for clarifying this point to me 
time is such that it uniquely and monotonically parameterises dynamical histories. It is holistic and democratic in exactly the sense that Barbour desires because it involves all the dynamical variables of a given system - crucially it is a measure of duration that 'emerges from the dynamics' and 'does not pre-exist in the kinematics' (Barbour (1994, p.2856)).

We can take the Hamiltonian formulation of Jacobi's principle as a model for the Hamiltonian formulation of any Machian temporally relational theory. In particular, it suggests a set of four criteria for the formal structure of such a theory: 1) the parameterisation of phase space curves is arbitrary; 2) the canonical variables do not contain external time variables or their momenta; 3 ) there is a Hamiltonian constraint that has a dynamical phase space action when combined with an arbitrary multiplier; 4) there exists a methodology for constructing an emergent temporal increment that parameterises dynamical histories in an equitable and unique manner. If 1-4 are satisfied, then it seems reasonable to accept that the theory admits an interpretation consistent with Machian temporal relationalism. As discussed above, such an interpretation has two key features: i) the absence of time in the basic ontological structure and ii) our ability to abstract an equitable measure of duration from the change (or relative change) of the objects that are part of the ontology. Specifically, it seems reasonable to assume that 1-2 lead to i) since they ensure that sequences of points within the phase space can be understood as representing the fundamental ontology without reference to time. We then have that 3-4 lead to ii) since, as illustrated by the case of Jacobi's principle, they give us the machinery to associate with pairs of points in the phase space (elements of the ontology) the appropriate temporal increment. The utility of our criteria (which are of a heuristic rather than logical character) is illustrated by their preclusion of a Machian temporal relationalist interpretation of parameterised particle mechanics (there 2 does not hold) and admission of such an interpretation for Barbour-Betotti theory (Barbour and Bertotti (1982)) (where 1-4 all hold).

Given these criteria, we can now address the task of evaluating the interpretation of canonical general relativity in terms of Machian temporal relationalism (MTR). ${ }^{15}$ Considering the action (10.3) leads us to conclude that the first and second conditions are satisfied and therefore to expect that aspect i) of MTR holds in canonical general relativity.

To an extent, we also have that the third condition holds because the Hamiltonian is of course a constraint and in one context its role is (when combined with the lapse) to generate a transition between dynamically related three geometries. However, as has been asserted throughout our discussion it is essential to remember that that Hamiltonian constraints of canonical gravity have a dual

\footnotetext{
${ }^{15}$ It must be noted here that much of Barbour's work on the Machian temporal structure of general relativity focuses on general relativity formulated in Lagrangian terms. Our focus on the canonical formalism will not obscure the essential aspects since they are inherent within the dynamical structure of general relativity and therefore beyond the Lagrangian/Hamiltonian distinction. See Pooley (2001) and Butterfield (2002) for detailed philosophical analysis using, for the most part, Barbour's version of the Lagrangian formalism.
} 
nature with two distinct, context-dependent roles. In the context of a hypersurface embedded within a solution, the role of the Hamiltonian constraints is not of the dynamical type found in Jacobi's theory. Rather, they generate infinitesimal symmetry transformations that form part of the hypersurface deformation group which manifests the fundamental symmetry of the theory. Still, this does not necessarily break the analogy between the relativistic and non-relativistic Hamiltonian constraints since in Jacobi's principle too the Hamiltonian constraint is also connected with unphysical temporal relabellings. However, the fact that the temporal relabellings associated with the infinite set of Hamiltonian constraints of canonical relativity are local and those associated with the single Hamiltonian constraint of Jacobi's principle are global is of crucial importance. Ultimately, the disanalogy that this subtle yet significant difference implies creates an acute problem for an interpretation of canonical general relativity in Machian temporally relational terms.

The fourth criterion that we introduced for the formal structure of MTR Hamiltonian theory was that we are able to construct an emergent temporal increment that parameterises dynamical histories in an equitable and unique manner. Given this together with the third criterion, it seems reasonable to presume that we can interpret the phase space of our theory to represent a Machian ontology in the sense of being amenable to the condition ii) above. More explicitly: if our theory is such that two distinct points in phase space that are dynamically related can be connected by the application of the Hamiltonian constraints times suitable multipliers and, furthermore, the difference between them is parameterised uniquely by an emergent time parameter, then we may interpret each point in the phase space as representing the state of the objects in the world and the change between these two distinct ontological states as encoding uniquely a measure of duration in terms of ontological change. In the case of canonical Jacobi's principle, we were able to satisfy this criterion through the employment of ephemeris time, and it is therefore natural to look to construct a similar emergent temporal increment to enable a Machian reading of canonical general relativity.

As has already been mentioned, the crucial difference between the two theories is that the single, global Hamiltonian constraint of Jacobi's principle becomes an infinite set of local Hamiltonian constraints in general relativity. Thus, rather than looking for a single global ephemeris time it is natural to look for many local ephemeris times. A point in the phase space of canonical general relativity corresponds to canonical data on a spacelike hypersurface $\sigma$. The locality of the ephemeris times is necessitated by the fact that we need one such time for every $x \in \sigma$. The lapse is of course itself a local function (the dependence on $x$ is suppressed in the notation above). Thus, by looking for formulation of the theory with a lapse such that, in analogy with (12.4), it defines the desired emergent temporal increment when multiplied by an infinitesimal change in the time parameter we can define our local ephemeris times. If we denote the lapse of such a formulation $N_{e m p h}(x)$, then the local ephemeris can be written simply as $N_{e m p h}(x) \delta t$, where $t$ is of course now playing the part of the arbitrary time parameter. 
The crucial problem is then finding a formulation of the theory containing a local ephemeris with the desired properties. In particular, as well as being insensitive to rescalings of the time parameter, we need our local ephemeris time to be such that it will replicate time as measured by local clocks and thus be consistent with proper time. Furthermore, it is also fundamental to the notion of Machian temporality that any given local ephemeris time be an equitable measure of duration, and therefore that it takes account of the contribution of all the other degrees of freedom - even those that are non-locally separated from the spatial point at which it is defined.

Interestingly, according to Barbour (2000) the 'deep structure' of general relativity already contains exactly the type of local ephemeris time that we are looking for. ${ }^{16}$ Starting with the BSW (Baierlein, Sharp, and Wheeler (1962)) reformulation of covariant general relativity one can derive (Barbour, Foster, and Murchadha, 2002, pp.10-12) an expression for the lapse that, within a Lagrangian picture, takes the form $N_{B S W}=\sqrt{T / 4 R}$ where $T$ is a 'kinetic energy' term (Barbour et al., 2002, (4.2)). If the time label $t$ within $T$ is chosen such that $N_{B S W}=1$ then $t$ will directly measure proper time. Furthermore, for arbitrary time label $N_{B S W}(x) \delta t$ will always be equal to the local proper time. Just as in the case of Jacobi's theory we can translate this Lagrangian emergent time framework into the a Hamiltonian analogue. There $N_{B S W}(x) \delta t$ gives us an emergent notion of duration that is equal to the local proper time calculated along the direction perpendicular to $\Lambda_{t}$ and is non-locally dependant upon the entire three-metric and its canonical momentum. Thus, local ephemeris time is a consistent notion within canonical general relativity (given the BSW formulation).

Furthermore, after the introduction of local ephemeris time we are able to classify pairs of points within dynamically successive (infinitesimally close) three-geometries as carrying a trans-temporal notion of identity. Such points are said to be equilocal, and the ephemeris time marks them out in terms of the unique temporal metric it provides. For our purposes, the crucial point is that the temporal metric that ephemeris time gives us is defined to be independent of arbitrary reparameterisations of the temporal parameter $(t$ in the case of canonical general relativity) and thus allows us to parameterise dynamical trajectories in phase space in exactly the manner required for criterion 4.

We thus have that 1-4 hold and would then expect canonical general relativity to admit a consistent interpretation in terms of Machian temporal relationalism. However, there is an acute problem with the Machian interpretation resulting, as foreshadowed above, from the locality of the Hamiltonian constraints. The necessary arbitrariness within the definition of $N_{B S W}(x)^{17}$ entails that given initial canonical data on a three geometry the dynamical evolution generated by $H\left(N_{B S W}\right)$ does not provide us with a unique continuation. This is

\footnotetext{
${ }^{16}$ Note: he does not use the phrase 'deep structure' in this quite this context!

${ }^{17}$ As noted in Barbour et al. (2002), although $N_{B S W}(x)$ is fixed on the initial slice by the initial data, its evolution is free
} 
the result of the Hamiltonian's second role of generating infinitesimal diffeomorphisms when considered in the context of hypersurfaces embedded in dynamical spacetimes. Two such spacetimes are of course represented by a pair of curves within the constraint surface in phase space. Let us assume that these curves are identical up to a given phase space point corresponding to canonical data on $\sigma_{0}$ and thereafter differ only in virtue of a different choice of the lapse - i.e., evolution generated by $H\left(N_{B S W}^{\prime}\right)$ rather than $H\left(N_{B S W}^{\prime \prime}\right)$. We should then consider them as only differing by a local temporal re-labelling, which (in spacetime terms) can be interpreted as an infinitesimal diffeomorphism of an embedded hypersurface. Given any point $x \in \sigma_{0}$, there will be an equilocal point within the subsequent three-geometries, $\sigma^{\prime}$ and $\sigma^{\prime \prime}$, associated with each of the distinct phase space curves. Thus, we run into exactly the problematic indeterminism discussed in $§ 4.2$. This problem does not occur in Jacobi's principle because the arbitrariness that remains within the lapse in that case only gains effect through a single global Hamiltonian constraint and thus cannot lead to distinct phase space curves. Thus, it seems that although an interpretation of canonical general relativity in terms of Machian temporal relationalism can be consistently achieved, this can be done only at the price of admitting ontological indeterminism into a theory that is manifestly deterministic in an empirical and, so far as the conventional interpretation of the covariant formulation goes, formal sense.

\subsection{Unsophisticated temporal relationalism and indeterminism}

Our discussion of $\S 4.1$ highlighted the concern that by treating points in the phase space of a gauge theory as representative of individual states we leave ourselves susceptible to a pernicious form of formal indeterminism within a physically deterministic theory. It should be no surprise therefore that, as we have defined it by the criteria 1-4, the Machian temporal relationalist approach to towards the Hamiltonian constraints and phase space of canonical general relativity leads to a specific case of exactly this kind of problem. In our earlier discussion we focused upon symplectic reduction as the supposed remedy for this indeterminism but found that in the case of canonical general relativity such a procedure has a trivialising effect. We are thus in need of an alternative, non-reductive methodology for dealing with indeterminism.

The indeterminism issue can be understood in terms of haecceitism. Again repeating the relevant material from Chapter 2 for the readers convienece. We define a haecceitist as someone who admits 'nonqualitative determinants of cross-identification' between entities or objects in distinct worlds or structures (Lewis (1983, p.19)). To adopt such a position is to allow for real differences that are only with respect to which objects play which role within the structure; since one is allowed to cross-identify each of a pair of qualitatively identical objects whose roles are permuted between two structures, we may ground a nonqualitative differentiation of the structures in terms of the cross-identification of the objects. The assumption that each point within the phase space of a gauge theory represents a distinct instantaneous state can be understood as an implicit endorsement of haecceitism. We can seen this since: i) We are licensed 
to consider as distinct two histories represented by sequences of points that differ solely with respect gauge transformations; ii) Such a difference is only with regard to which instantaneous states (represented by points) play which roles; iii) This means that if we take a history to be the relevant structure and instantaneous states (labelled by the points to which they correspond) to be the relevant objects, then the ontological difference between gauge related histories can be cashed out in haecceitist terms. By moving to a reduced phase space, we allow for the maintenance of haecceitism without indeterminism. However, conversely by disavowing haecceitism we can maintain ontological determinism without reduction. This is evident since an anti-haecceitist position would involve discounting the possibility of differences between two structures or worlds that do not have any qualitative differences - we do not allow differences only as to which objects play which role within a given structure. Thus, by antihaecceitist lines two gauge related curves within a phase space must be taken to represent the same ontological object.

In the context of spacetime points, anti-haecceitism has been employed towards the endorsement of both relationalism and the opposing position of substantivalism. According to the anti-haecceitist substantivalist (the 'sophisticated substantivalist'), spacetime points are real entities cannot be non-qualitatively cross-identified across models. Thus, even if we accept that diffeomorphically related spacetimes in covariant general relativity do not constitute distinct physical possibilities, this does not mean that we must accept spacetime points as non-fundamental. Rather, the sophisticated substantivalist can take an individual spacetime to be multiply realised within the equivalence class (as constituted by the relevant orbit of the four-dimensional diffeomorphism group) and thus maintain the fundamental status of the constituent spacetime points in terms of this multiple realisation. This anti-haecceitist strategy is of course also available to someone who wishes to exclude spacetime points from their basic ontology since it allows one to avoid exactly the Leibniz equivalence violating modal excess that individuated spacetime points leads to. The key to relationalism of this sort is that the fundamental ontology does not include spacetime points, and there is clearly no conflict between this position and anti-haecceitism. Thus we may avoid indeterminism, reduction and spacetime points by endorsing an anti-haecceitist relationalist position, which we shall follow Rickles (2007) and categorise it under the mildly pejorative moniker of unsophisticated relationalism.

As before, our object is not to review the substantial literature on spacetime relationalism/substantivalism but rather to utilise the ideas of this literature in the specific case of time within canonical general relativity with which we are occupied. In particular, it is extremely interesting to determine if we can make use of anti-haccestism to relieve the Machian temporal relationalism from the spectre of indeterminism. Essentially, an unsophisticated temporal relationalist (UTR) is going to disavow the reality of local temporal labellings and endorse the notion that spacetimes related by local relabellings (i.e., re-foliations) are single individuals multiply realised in terms of equivalencies classes. In the canonical context, this equates to treating phase space curves that are equiva- 
lent to re-foliations of the same spacetime as representing the same fundamental ontology. This is not equivalent to treating the phase space action of the Hamiltonian constraints as generating gauge equivalence classes - such a position is, as we have seen, problematic and manifestly distinct from both the relationalist/substantivalist and haecceitist/anti-haccestist disjuncts. Rather our unsophisticated temporal relationalist, unlike the reductive temporal relationalist, can account for the dual role of the Hamiltonian constraints by, on the one hand, treating the curves it generates in phase space as dynamics and, on the other, by classifying the two such curves that are related purely by the deformation of a constituent three-geometry as representing the same basic object (multiply realised). It is very important to note that the equivalence classes in question do not have a direct representation on the phase space. Essentially, they are four- and not three-dimensional objects because re-foliations are symmetries at the four- and not three-dimensional level. Given a spacetime (and the associated phase space path), we can consider the equivalence class of other spacetimes (and associated phase space paths) related by re-foliations. Thus, although we are still to some extent operating on the level of phase space, in order to construct the well defined notion of equivalence class that we need to ground our position, we must always consider three-geometries in the context of embedding within a spacetime. We do not have a mechanism for classifying individual three-geometries as realised multiply in terms of a gauge equivalence class. Rather it is the individual four-geometry that is multiply embodied by gauge related collections of three-dimensional objects.

This last and crucial point makes it clear that the kind of unsophisticated temporal relationalist position which we have outlined is going to make an uncomfortable bedfellow for the Machian notion of relationalism. UTR as we have defined it essentially makes use of an ontology predicated upon four-dimensional spacetimes and not merely sequences of three-geometries. The relevant equivalence class defining the symmetry relation can only be properly defined in the spacetime context. Thus, we violate the key MTR notion that time (or spacetime) should not form part of the basic ontological structure. UTR does allow for a viable notion of relationalism (to the extent of allowing us to exclude temporal points) and would seem to be compatible with the emergent notion of time that forms the other key aspect of the Machian position. However, it is essentially a spacetime theory of temporal relationalism and thus cannot be construed as Machian in the most fundamental sense.

\subsection{Scale invariance and Machian temporal relationalism}

We thus return to the dilemma of extricating the Machian temporal relationalist philosophy from the ontological indeterminism issue. As was mentioned above, the root of the problem lies within the local nature of ephemeris time and this in turn is due to foliation invariance. It is therefore fairly obvious that a solution could lie within the fixing of a foliation and with, therefore, a Machian temporally relationalist interpretation of canonical general relativity in a preferred foliation. Three issues with such a strategy are immediately apparent. First, there must be a basis for this preferred foliation that is, at the very least 
non- ad hoc, and preferably driven by Machian underpinnings. Second, if we are to exclude large sectors of the traditional (canonical) solution space by fixing a foliation, then those solutions excluded must be at the very least not empirically grounded, and preferably not empirically viable. Third, the foliation-fixed version of canonical GR must still be consistent with the Machian criteria 1-4 introduced above. Recent years have in fact seen dramatic improvements for the provision of good answers to all three of these points through the development of a scale invariant approach to Machian general relativity know as shape dynamics. We do not have space here to give a detailed introduction to or description of this programme and its recent developments. We can at least, however, give a basic outline of its key elements such that we can consider shape dynamics in the context of the three points regarding foliation fixed canonical GR and MTR just raised.

As philosophical and methodological attitude taken towards physical theory, the Machian approach is one that in general advocates the elimination of absolute or background structure. Modern Machians, Julian Barbour of course being most notable amongst them, argue that whether in Newtonian mechanics or general relativity such structure should be cleaved from our representation of the world via the adoption of alternative, appropriately minimal, theories of mechanics. In this sense, Machianism can be seen as a general scheme for eliminating absolute structure, minimising initial data, and a description of the world based in some sense on relations. This general programme should not be conflated with the specific projects of Machian temporal and spatial relationalism. One would hope, however, that the two cohere - and with regard to absolute structure relating to scale and time so it appears to be the case.

There is within all the major theories of mechanics, including general relativity, an absolute notion of scale - conformal transformations (i.e., those which preserve angles but not lengths) are not symmetries at either the local or global level. Within covariant general relativity, this means that solutions of the theory are not invariant under conformal transformations of spacetime. Attempts to construct a gravitational theory that is $4 \mathrm{D}$ conformally invariant have a long history stretching back to Weyl (1922). More pertinent to our project is the programme of constructing a 3D scale invariant theory - i.e., one that is invariant under conformal transformations of space. The investigation of implementing such a symmetry within general relativity in fact parallels the development of the canonical approach in that it can also be traced back to the late fifties and Dirac (1959). In both this work and its extension by York (1973), we already have 3D conformal invariance explicitly connected to a gauge-fixed formulation of general relativity with a preferred foliation. More, recently Gomes, Gryb, and Koslowski (2011) have build on the work of Barbour and Murchadha (2010) and Anderson, Barbour, Foster, Kelleher, and Murchadha (2005) to propose the existence of a intrinsic duality between a theory invariant under volume preserving local 3D conformal transformations and general relativity. Specifically, the particular gauge fixing of general relativity that corresponds to the foliation of spatially compact spacetimes into space-like hypersurfaces of constant mean curvature (the CMC gauge) is taken to be equivalent to a particular 
gauge fixing of a 'dual theory' that describes sequences of spatial three manifolds invariant under both three-dimensional diffeomorphisms and 3D (volume preserving) conformal transformations. Crudely and yet fairly accurately put, the essence of this shape dynamics programme is then to exchange the foliation symmetry which is present in GR for the local conformal symmetry which is absent. Thus, we can provide a reasoned and, what is more, Machian basis for fixing a foliation.

Our second concern above was that since fixing a foliation amounts to a restriction to a particular sector of the solution space of general relativity there is a danger that it might have undesirable consequences with regard to the empirical adequacy and/or predictive power of the theory. This concern is directly analogous to that discussed in $\S 4.2$ regarding the restriction to globally hyperbolic solutions that is entailed by moving to the canonical formalism. In essence, so far as it relates to canonical general relativity, the shape dynamics approach amounts to the introduction of the restriction that as well as being globally hyperbolic solutions must be CMC foliable. According to Gomes et al. (2011) this is a weak restriction since it 'includes the vast majority of physically interesting solutions to Einstein's equations while excluding many physically uninteresting solutions'. Thus, one may be able to argue that empirically nothing has been lost - certainly we are able to retain the solutions most relevant to currently observed empirical phenomena since the Schwarzschild, FRW, Reissner-Nordström and Kerr-Newman solutions are all CMC foliable (at least so long as we exclude the areas within the event horizon of black hole solutions). Furthermore, as was argued above for the canonical general relativity and the hyperbolic solution case, we are not invoking an ad-hoc philosophical principle in order to exclude these solutions but rather a theory derived from definite physical principles (in this case 3D scale invariance). A more forceful response to this worry is to convert this supposed empirical deficiency into a prediction. Since the restriction to CMC foliable spacetimes can be seen as a consequence of shape dynamics, we may argue that it is providing us with a falsifiable statement about the world that goes beyond those provided by conventional general relativity. Additionally, it also in a sense offers us an explanation why our universe does not manifest phenomena relevant to non-CMC foliable solutions if they are nomologically possible, why do we not find them or approximations to them in nature? Admittedly, as independent arguments for preferring shape dynamics over traditional general relativity these are not altogether convincing lines of reasoning, but their adoption certainly seems enough to blunt any criticism of the approach along the same lines.

Our third, and most important, worry concerning foliation fixing and MTR is whether general relativity, so formulated, still has the necessary characteristics 1-4 that were deemed necessary for a theory to be susceptible to the relevant Machian relational interpretation of temporality. To investigate this point in the context of shape dynamics, we must consider the latter in a little more technical detail. The methodology for constructing the scale invariant 'dual theory' that Gomes et al. (2011) employ can be broken down into five distinct stages. We will briefly outline these in order to argue that the resulting theory can be 
understood in terms of the notion of Machian temporal relationalism that we have introduced. The first step is to explicitly identify the requisite symmetry that will be exchanged for foliation invariance. This is the quotient group denoted by $\mathcal{C} / \mathcal{V}$. Here $\mathcal{C}$ is the (Abelian) group of conformal transformations on the (assumed to be compact) spatial three manifold, which in our notation is $\sigma$. The elements of this group are scalars $\phi: \sigma \rightarrow \mathbb{R}$ which are such that:

$$
\begin{array}{r}
q_{a b}(x) \rightarrow e^{4 \phi x} q_{a b}(x) \\
P_{a b}(x) \rightarrow e^{-4 \phi x} P_{a b}(x)
\end{array}
$$

$\mathcal{V}$ is then a one parameter sub-group representing homogenous conformal transformations. The explicit construction of $\mathcal{C} / \mathcal{V}$ in terms of equivalence classes of conformal transformations $[\phi]$ then enforces that there exists a unique representative which leaves the three volume $V_{q}=\int_{\sigma} d^{3} x \sqrt{|q|}(x)$ invariant (see Eq. 61 and the surrounding discussion of Gomes et al. (2011) for details). This then allows us to parameterise the group $\mathcal{C} / \mathcal{V}$ by scalars associated with volume preserving conformal transformations and thus indicates that we have identified the appropriate symmetry group. The next step is to formally adjoin this symmetry to the theory. Glossing over the technicalities of exactly how this is done (see Gomes et al. (2011, §4.1.2)), we can understand this stage in terms of an extension of the phase space of canonical general relativity through the introduction of additional canonical variables (the Stückelberg field and its conjugate momenta), which in turn, due to dynamical consistency requirements, results in the presence of an additional set of first class constraints $C(x)=0$. Like the Hamiltonian constraints, there is one of these constraints per spatial point. However, unlike the Hamiltonian constraints the $C(x)$ can be straightforwardly understood as generating unphysical gauge transformations (akin to the transformations generated by the momentum constraints) - these are the volume preserving conformal transformations. Importantly, because of the fact that they are volume preserving one of the new constraints reduces to an identity, so in fact there is one conformal constraint less then there are Hamiltonian constraints. The third step is to impose a gauge fixing via a best matching procedure (see Gomes et al. (2011, §4.1.3) and references therein) such that all but one of the original Hamiltonian constraints becomes second class (in the sense of the standard Dirac (1964) terminology). The usual dynamical consistency conditions of the Dirac prescription for dealing with second class constraints leads to a particular fixing of the lapse up to a one parameter freedom. This lapse fixing is precisely that which gives the equivalence class of CMC foliations. Still following the Dirac procedure, it is possible to eliminate the second class constraints (Gomes et al. $(2011, \S 4.1 .4)$ ) and arrive at a theory with a Hamiltonian that is constituted by the sum of three distinct types of first class constraint combined with the appropriate multipliers. This new theory is shape dynamics, and its relationship with canonical general relativity is such that for a specific gauge fixing it is equivalent to canonical general relativity in the CMC gauge. Like canonical general relativity, both the symmetry and dynamical properties of the theory are encoded within the structure of the different types of con- 
straints. The first of these constraints are the conformal constraints, which are responsible for the theory's invariance under volume preserving conformal transformations. Next are momentum constraints, which although they have been transformed in the passage to the new phase space can still be understood as implementing three-dimensional diffeomorphism invariance as in the original theory. Finally, and most important for our purposes, there is a single Hamiltonian constraint. This constraint is exactly analogous to the single Hamiltonian constraint of Jacobi's principle: it generates dynamics when considered as acting on phase space and global reparameterisations when considered as acting on an entire solution.

Let us now consider our four criteria for a theory to be susceptible to an interpretation in terms of Machian temporal relationalism. Within the dual theory, the parameterisation of phase space curves is arbitrary (i.e. 1), and furthermore the canonical variables do not contain external time variables or their momenta (i.e. 2). We can also now see that, since there is a Hamiltonian constraint that has a dynamical phase space action when combined with an arbitrary multiplier, we also have 3 . Thus the condition for an interpretation in terms of MTR is that there exists a methodology for constructing an emergent temporal increment that parameterises dynamical histories in a equitable and unique manner (i.e. 4). Since we have a single Hamiltonian constraint which is combined with a special lapse with a one parameter freedom, intuitively it seems that the construction of the requisite notion of global ephemeris time should be possible within the dual theory itself. However, sidestepping the interesting technical challenge of explicitly constructing such an object, we can, because of the duality between the theories, consider instead the parallel issue within CMC foliated canonical general relativity. Here it transpires our problem is in fact effectively already solved since it has long been know that all spacetimes admitting a CMC foliation can be parameterised by a unique geometric time (See Belot (2007, §7.3) for discussion of the details). Since it is determined by the difference in intrinsic curvature between slices in a dynamical solution this geometric time is both unique and suitably equitable. Thus, almost by definition, canonical general relativity in the CMC gauge satisfies our condition 4. We can therefore assert that both this form of general relativity and the dual theory are amenable to an interpretation in terms of MTR.

\section{Denial III: Complete Observables and The Parmenidean State}

We now turn to our third denial of time which is based upon Rovelli's complete and partial observable scheme (Rovelli $(1990,1991,2002,2004)$ ) as applied to canonical general relativity within the work of Dittrich $(2006,2007)$ and Thiemann (2007). The notion of temporality that is implied by this scheme could be conflated with our first denial in term reductive temporal relationalism. However, as we shall see, despite some superficial similarity with regard to how the Hamiltonian constraints are treated there are in fact deep conceptual differences. In particular, whereas reductive relationalism is predicated upon the reduced phase space, the Rovelli-Dittrich-Thiemann (RDT) approach is unequivocally 
non-reductive. Furthermore, whereas reductive temporal relationalism, and for that matter relationalism in general, is fundamentally a thesis with regard to the priority of relational over purely temporal structure, the RDT approach can only naturally be interpreted in terms of a philosophical framework which precludes temporal structure altogether. We will begin our discussion of this third denial by first introducing the RDT scheme in the context of the simple nonrelativistic case of Jacobi's theory.

\subsection{The Complete and Partial Observables Ansatz}

Consider the physical phase space of Jacob's theory, $\Gamma_{J}=\left\{(p, q) \in \Pi_{J} \mid H(p, q)_{J}=\right.$ $0\}$, which is a sub-manifold within the extended phase space defined by the satisfaction of the constraint. According to standard Dirac machinery for dealing with constrained Hamiltonian theories we define as the observables the class of functions on this physical phase space which have vanishing Poisson bracket with the constraints. With the weak inequality implying restriction to the constraint surface, we can write this as a condition of a general extended phase space function $f: \Gamma_{J} \rightarrow \mathbb{R}$ as $\left\{f, H_{J}\right\} \approx 0$. Like with reduction with respect to Hamiltonian constraints in general relativity the application of this standard definition has immediate, and problematic, consequences for our description of change. If the observable functions must commute with the Hamiltonian then they must also be non-changing along dynamical trajectories. It seems that either: i) this definition of observable; or ii) our expectations for the notion of change that our theory provides us, must be adapted to deal with theories in which the Hamiltonian is a constraint. The essence of the RDT both as it applies to Jacobi's theory and general relativity is to assert that the problem lies within i). With some ingenuity we can construct observable functions with nontrivial representational capacity so along as we abandon the notion that these obervables change in any conventional sense. The proposal for constructing such obervables is what we shall call the complete and partial obervables Anstaz and we shall introduce it here first in terms of the Dittrich (2007) nonrelativistic treatment.

First let us label the configuration variables within phase space partial observables and the designate the specification of relations between these variables complete observables. The latter are constituted with Jacobi's theory by the reparameterisation invariant specification of the value of one configuration variable with respect to another - as correlations between partial observables. The complete observables are the families of correlation functions which individually give the value of one of the partial observables when the other (the clock variable) is equal to some real number.

Consider a system described by two configuration variables (partial observables) $q_{1}$ and $q_{2}$ which together with their conjugate momenta obey a Hamiltonian constraint of the form $H\left[q_{1}, q_{2}, p_{1}, p_{2}\right]=0$. The phase space, $\left(q_{1}, q_{2}, p_{1}, p_{2}\right) \in \Gamma$, will as usual have a symplectic structure. We can use the relevant symplectic form to define the action of the Hamiltonian vector field on an arbitrary function, $X_{H}(f)=\omega\left(X_{f}, X_{H}\right)=\{f, H\}$. The flow, $\alpha_{H}^{\tau}$, generated by this vector field can then be defined for every $x \in \Gamma$ and we can see this flow 
as acting on a phase space function, $\alpha_{H}^{\tau}(f)(x)$, such that it takes the function along the solution. ${ }^{18}$ For our system therefore we calculate $\alpha_{H}^{\tau}\left(q_{1}\right)\left(q_{1}, q_{2}, p_{1}, p_{2}\right)$ and $\alpha_{H}^{\tau}\left(q_{2}\right)\left(q_{1}, q_{2}, p_{1}, p_{2}\right)$ We then designate one of our variables as a clock variable and seek to invert an expression of the form $T_{x}(\tau)=\alpha_{H}^{\tau}\left(q_{1}\right)(x)$ such that solving $T_{x}(\tau)=s$ for $s \in \mathbb{R}$ will give us an expression for $\tau$ in terms of $s$ and $q_{1}$. In general this inversion will only be possible for a specific interval - thus the clock variables are typically going to be at best locally well defined and so are unlikely to be continuous on phase space and this means that the scheme will be difficult to implement in practice. We can then insert the inverted expression into the second flow equation $\alpha_{H}^{\tau}\left(q_{2}\right)(x)$ by substituting for $\tau$, and produce an expression which (within the interval specified) gives us the value of $q_{2}$ when $q_{1}$ takes the value $s$. This complete observable represents a family of functions (one for each $s$ ) each of which expresses the correlation between our two partial observables without reference to parameterisation.

Importantly, the conceptual leeway to consider a family of these complete observables rather than a single correlation is dependent on the use of the unreduced formalism. Thus, even though we are in a sense utilising the standard Dirac condition for observable function we are not thereby committing to the passage to the reduced phase space that is generally assume to go along with it - we are only being consistent with Dirac observables scheme so far as it relates to the unreduced phase space. This makes explicit the difference between this approach and reductive relationalism. It also implies that unlike functions of the reduced phase space complete observables have non-trivial representational capacity since within a given family of observables we may represent the physical structure of a dynamical universe.

\subsection{Application to General Relativity}

Application of the complete and partial obervables Anstaz to canonical general relativity poses a challenge of far greater difficulty for several reasons. We of course have many and not one constraint and in order to be a true complete observables the object we construct must therefore be constant along the flow associated with all constraints. If all the constraints were mutually Poisson commuting and finite in number this could be addressed by the technically difficult, but conceptually fairly straight forward, process of: i) introducing one clock variable per constraint; and ii) considering as our complete observable a product between each of the flows generated by each of the constraints when applied to a given partial observables, as evaluated for a specific value of each of the relevant flow parameters. We would then have a family of complete observables which were closely analogous to those consider above for Jacobi's theory, only they are now constant along all the various gauge orbits. However, canonical general relativity of course has an infinite number of constraints and, what is more, these constraints do not Poisson commute. As pointed out by Thiemann (2007), even if we restrict our selves to the space of spatially

\footnotetext{
${ }^{18}$ See Dittrich (2007, Eq. 2.5-7) for explicit formulas.
} 
diffeomorphism-invariant functions (i.e., those satisfying $\{\vec{H}(\vec{N}), f\}=0$ ) a flow which is associated with a given Hamiltonian constraints and acts on such a function will not itself be spatially diffeomorphism-invariant since the bracket $\{\vec{H}(\vec{N}), H(N)\}=-\kappa H\left(\mathfrak{L}_{N_{a}} N\right)$ is not invariant. Moreover, even if we remove the momentum constraints altogether and presume ourselves to be working in superspace we still have to deal with the even more highly non-trivial Poisson bracket between the Hamiltonian constraints which features structure functions. Thus, the application of the basic RDT scheme outlined above to canonical general relativity poses a significant challenge.

Encouragingly, a number of proposals for meeting this challenge have been put forward. One is that of Dittrich (2006), ? which gives an explicit demonstration of how complete observables for general relativity may be constructed in stages by first computing partially compete observables which are complete observables with respect to a sub-algebra of the constraints and then using these objects to to calculate complete observables with respect to all the constraints. The partial observables in this construction are constituted by spacetime scalars which in turn are constructed out of canonical fields, and this process serves to reduce the number of constraints that must be dealt with. For reasons of space we will not here attempt a explanation of the details of the Dittrich approach but rather turn our attention to an alternative methodology know as the master constraint programme. The choice between these two approaches is far from a trivial one and particularly with regard to quantisation it may have significant technical implications. However, for our purposes it must be noted that in respect of the interpretational implications with regard to time the fundamental features are common to both methodologies and we are choosing to focus on the second only because it may be introduced more concisely.

In general the idea of the master constraint programme (Thiemann (2006, $2007)$ ) is to re-write constraint functions, $\varphi_{j}(p, q)=0$, in terms of a single equation which will be satisfied under the same conditions. This new single constraint is then the master constraint. A simple example is given by taking a positive quadratic two form $K^{i j}$ and constructing the equation:

$$
\mathbf{M}:=K^{i j} \varphi_{i} \varphi_{j}=0
$$

This equation is satisfied if and only if all the individual constraint functions are vanishing and thus defines the same physical phase space $\Sigma$ that we had before. An observable condition for the extended phase space is then given considering the class of functions such that:

$$
\left.\{\{\mathbf{M}, \mathcal{O}\}, \mathcal{O}\}\right|_{\mathbf{M}=0}=0
$$

i.e. those functions which have a vanishing double Poisson bracket with the master constraint on the constraint surface. Strictly, this is a restriction that implies that the observable functions generate finite symplectomorphisms which preserve $\Sigma$, rather than the usual Dirac condition that the observables are constant along the null directions generated by the individual constraints. However, it can be demonstrated that the two conditions are equivalent Thiemann (2006). 
For canonical general relativity the explicit form of the master constraint is given as:

$$
\mathbf{M}=\frac{1}{2} \int_{\sigma} d^{3} x \frac{H(x)^{2}}{\sqrt{\operatorname{det}(q)}(x)}
$$

This constraint has a number of formal virtues, in particular it is such that its satisfaction implies that $H(N)=0$ for all $N$ meaning that encodes the same constraint surface as the Hamiltonian constraints. Furthermore, it is also such that $\{\vec{H}(\vec{N}), \mathbf{M}\}=0$ meaning that it is invariant under spatial diffeomorphisms and will lead us to a constraint algebra with a much simpler form; the master constraint algebra, $\mathfrak{M}$ :

$$
\begin{aligned}
\left\{H_{a}\left(N_{a}\right), H_{a}\left(N_{a}^{\prime}\right)\right\} & =-\kappa H_{a}\left(\mathfrak{L}_{N_{a}} N_{a}^{\prime}\right) \\
\left\{H_{a}\left(N_{a}\right), \mathbf{M}\right\} & =0 \\
\{\mathbf{M}, \mathbf{M}\} & =0
\end{aligned}
$$

We no longer have to deal with the presence of structure functions in our constraint algebra since the highly complex expression (Eq. 10) in the Dirac algebra is replaced by the trivial self-commutation expression above. In substituting a single master constraint for the infinite set of Hamiltonian constraints we avoid having to explicitly confront the difficulties of the Poisson bracket algebra with which the latter are associated. Assuming the momentum constraints have been dealt with, either through reduction or via the Dittrich methodology mentioned above, we could now proceed to construct complete observable with respect to single master constraint by considering the flow $\alpha_{\mathbf{M}}^{\tau}$. A family of complete observables is then constituted the one parameter set of functions defined by the value of one partial observable when the other takes the value $s$. Assuming these functions are continuous a given complete observables can then be taken to be invariant under the simultaneous phase space transformations generated by all the Hamiltonian constraints taken together. Thus, as in the case of Jacobi's theory we arrive at an objects which is defined such that it is constant along the dynamical trajectory associated with the relevant 'gauge orbit' - but which has non-trivial representational capacity because it is part of a family of such functions defined within the unreduced formalism. This strange temporal structure is the hallmark of compete observables when applied to the case of Hamiltonian constraints and we now turn to the consideration of the associated interpretation implications for the nature of time.

\subsection{Physical States as Timeless Histories}

In our discussion of the Hamiltonian constraints of canonical gravity in $\S 2.3$ we emphasised the necessity of treating the constraints such that both the fundamental symmetry of the theory and dynamics are respected. The problem of triviality that beset the reductive temporal relational stance can be understood as a failure on the second count and the problem of indeterminism that troubled the Machian temporal relationalist stance (sans a fixed foliation) can 
be understood as a failure on the first. The kernel of brilliance that allows the RDT scheme to avoid both of these problems is to construct the families of complete observables such that the specification of each family member is deterministic, since they are individually constant along the orbit associated with the Hamiltonian constraints, and yet collectively they are still adequate to represent dynamical universes because of the use of the unreduced phase space. Thus, by endorsing the complete observables as our fundamental object we are provided with an ontology which solves at least one aspect of the problem of time in canonical gravity. However, unlike in a shape dynamics implementation of Machian temporal relationalism, we are not provided with a notion of how to represent change. In the case of our second denial, although time is absent in the sense that it has been relegated to an emergent level, it is still a substantive notion as derived concepts. Moreover, change is still a well defined notion, as we are free to specify the evolution of observable quantities between hypersurfaces with respect to an ephemeris time. What notion of change can we attach to the RDT scheme?

In order to answer this question it is instructive to consider certain key remarks of the three physicists themselves. In discussion of the nonrelativistic application of the complete and partial observables scheme Rovelli (2002) distinguishes the 'physical phase space' as the 'space of orbits generated by the constraints on the constraint surface' (p.3). In a similar vain Dittrich (2007) defines the physical state as an 'equivalence class of phase space points' which 'can be identified with an $n$-dimensional gauge orbit' (p1894). So far as they apply to the Hamiltonian constraints as considered acting on the phase space of canonical general relativity such a notion of 'physical space space' and 'physical state' imply an equation between the concept of a history and the concept of a physical state which is radically discontinuous with conventional mechanical theory. Typically states are taken to be instantaneous configurations and histories sequences of such states. In standard gauge theories, where the constraints can be understood unproblematically as generating unphysical transformations, phase space points connected by a gauge orbit are classified as the same state because the difference between them is taken to be unphysical. Dynamical histories are then constituted by either curves within the unreduced phase space which are no where parallel to these orbits or, more simply by curves, within the reduced phase space. Following the remarks of Rovelli and Dittrich above, the interpretation of change within the complete observables scheme still leads us to classify two points on a 'gauge orbit' as the same state; however this is because the word 'state' is redefined such that in includes all points on the orbit. For the case of the Hamiltonian constraints of general relativity this is simply to adopt a notion of state that involves no temporal specification at all, but rather implies that the observables of a theory are smeared everywhere along entire histories. Put more precisely, the complete observables can be understood as 'completely non-local in the unphysical time' (Thiemann (2007, p.81)).

The only viable interpretation of the RDT scheme as applied to genera relativity is then one in which time and change have no part - not even at an emergent level. This is to adopt a starkly Parmenidean view - time is purely an 
illusion - and thus constitutes a denial of time in a much thicker sense than that involved in Machian temporal relationalism. It is, however, unlike that involved in reductive temporal relationalism, a coherent denial. We may still describe dynamically nontrivial universes within the formalism but almost paradoxically we are able to do this whilst disavowing change. The key to untying this seemingly Gordian conceptual knot is that although individual complete observables are eternally frozen, within the families of such observables - which can only exist because we have avoided reduction - we have access to additional conceptual equipment which allows for the representation of universes corresponding to dynamical spacetimes. This brings us to an important qualificatory remark regarding our third denial of time. The Parmenidean position with regard to change that is forced upon us by RDT scheme does not equate to a denial of time either in the sense of asserting that there exists only one time. Nor is it a position which implies that the temporal dimension is less fundamental than the spatial dimensions - in of itself it is entirely consistent with a four dimensional spacetime picture of the world. We can therefore see that rather than being allied to the Machian notion of timelessness of our second denial, this third denial is in fact in some ways antithetical to it. In particular, if we were to couple the application of the complete observables scheme to the Hamiltonian constraints with an application of the scheme to the momentum constraints as well, then the resulting doubly complete observables will be objects smeared non-locally in the unphysical spacetime coordinates and this is an ontology which clearly is not amenable to the Machian temporally relationalist interpretation since it is predicated upon a fundamentally four rather than three dimensional picture of reality. Thus, the choice between our two denials is effectively that between: i) loosing four dimensionality and absolute scale but retaining change; and ii) retaining absolute scale and four dimensionality but loosing change.

\section{Conclusion}

Our analysis of the temporal structure of canonical general relativity and its various interpretations has rested upon the need to respect the fundamentally dual role of the Hamiltonian constraints that occur within the theory. Any approach towards the formal structure of the theory must take into account the role of these constrains in generating dynamics, in the context of phase space, and of generating unphysical symmetry transformations, in the context of a hypersurface embedded within a solution. It is in this respect that the first of our three denials of time was found to be acutely deficient, and it is because of this deficiency that the position of reductive temporal relationalism can only be found to be dynamically trivialising. It is also due to a failure to respect the the duality of roles played by the Hamiltonian constraints that our second denial was hampered by the problem of indeterminism, and, according to our analysis, it is only by removing the symmetry generating side of the Hamiltonian constraints and fixing a foliation that the second denial can remain consistent. The third and final denial of time is the starkest, and most subtle. Through implementation of the complete observables scheme within canonical general 
relativity we are led inevitably towards disavowing change and therefore time, and yet it seems that we may also maintain the conception of the world is terms of some aspect of the four dimensionality of a spacetime approach.

Between the two interpretations of canonical general relativity that are allied to the application of Rovelli-Dittirch-Thiemann scheme and the scale invariant implementation of Machian temporal relationalism there are clearly key formal and philosophical differences. However, the choice between these approaches, and therefore their resulting interpretations, is as yet in want of a strong technical or empirical basis. What would seem the best candidate for a criterion to break this interpretational underdetermination must be the prospect that each provides for a canonical quantisation of general relativity. Thus, the last word in the interpretation of the temporal structure of canonical general relativity will

probably in the end come from within a solution to the infamous challenge of constructing a quantum theory of gravity. Given our current state of ignorance, it perhaps best behoves us to accept; upon that which we cannot as yet sensibly speak of, we should remain silent.

\section{Acknowledgments}

Thanks to Julian Barbour, Hans Westman, Sean Gryb, Dean Rickles and Peter Evans for discussions/comments relating to this paper. I am also appreciative of the feedback on various versions of this material given by audiences in Sydney, Aberdeen, Dublin and Oxford.

\section{References}

Anderson, E., Barbour, J., Foster, B. Z., Kelleher, B., Murchadha, N. Ó., May 2005. The physical gravitational degrees of freedom. Classical and Quantum Gravity 22, 1795-1802.

Arnold, V. I., Kozlov, V. V., Neishtadt, A., 1988. Mathematical aspects of celestial mechanics. Springer-Verlag.

Arnowitt, R., Deser, S., Misner, C. W., 1962. The Dynamics of General Relativity. In: Witten, L. (Ed.), Gravitation: An Introduction to Current Research. John Wiley \& Sons Inc., New York, Ch. 7, pp. 227-265.

Baierlein, R. F., Sharp, D. H., Wheeler, J. A., 1962. Three-Dimensional Geometry as a Carrier of Information about Time. Phys. Rev. 126, 1864-1865.

Barbour, J., 1994. The timelessness of quantum gravity: I. The evidence from the classical theory. Class. Quant. Grav. 11 (12), 2853-2873.

Barbour, J., 1995. General Relativity as a Perfectly Machian Theory. In: Barbour, J. B., Pfister, H. (Eds.), Mach's Principle: From Newton's Bucket to Quantum Gravity. pp. 214-236.

Barbour, J., 2000. The end of time, paperback Edition. Oxford University Press. 
Barbour, J., 2009. Mach's principle, general relativity and gauge theory (unpublished draft).

Barbour, J., Foster, B., Murchadha, N. O., 2002. Relativity without relativity. Classical and Quantum Gravity 19, 3217.

Barbour, J., Foster, B. Z., Aug. 2008. Constraints and gauge transformations: Dirac's theorem is not always valid. ArXiv e-prints.

Barbour, J., Murchadha, N. Ó., Sep. 2010. Conformal Superspace: the configuration space of general relativity. ArXiv e-prints.

Barbour, J. B., Bertotti, B., 1982. Mach's principle and the structure of dynamical theories. Proceedings of the Royal Society of London. A. Mathematical and Physical Sciences 382 (1783), 295-306.

Belot, G., 1999. Rehabilitating relationalism. International Studies in the Philosophy of Science 13, 35-52.

Belot, G., 2000. Geometry and motion. British Journal for the Philosophy of Science 51, 561-95.

Belot, G., 2003. Symmetry and gauge freedom. Studies In History and Philosophy of Modern Physics 34, 189-225.

Belot, G., Jan 2007. The representation of time and change in mechanics. In: Butterfield, J., Earman, J. (Eds.), Handbook of Philosophy of Physics. Elsevier, Ch. 2.

Belot, G., Earman, J., 1999. From metaphysics to physics. In: Butterfield, J., Pagnois, C. (Eds.), Fron Physics to Philosophy. Cambridge University Press, pp. $166-186$.

Belot, G., Earman, J., 2001. Pre-socratic quantum gravity. In: Callender, C., Hugget, N. (Eds.), Physics Meets Philosophy at the Planck Scale. Cambridge University Press.

Bergmann, P. G., 1961. Observables in General Relativity. Rev. Mod. Phys. 33, $510-514$.

Butterfield, J., 2002. Critical notice. The British Journal for the Philosophy of Science 53 (2), 289-330.

Dirac, P. A., May 1959. Fixation of Coordinates in the Hamiltonian Theory of Gravitation. Physical Review 114, 924-930.

Dirac, P. A. M., 1958. The Theory of Gravitation in Hamiltonian Form. Proceedings of the Royal Society of London. Series A, Mathematical and Physical Sciences 246, 333-343.

Dirac, P. A. M., 1964. Lectures on quantum mechanics. Dover Publications. 
Dittrich, B., 2006. Partial and complete observables for canonical general relativity. Classical and Quantum Gravity 23, 6155.

Dittrich, B., 2007. Partial and complete observables for Hamiltonian constrained systems. General Relativity and Gravitation 39, 1891.

Earman, J., 2002. Thoroughly modern mctaggart: Or, what mctaggart would have said if he had read the general theory of relativity (2002). philosophers' imprint. Philosopher's Imprint 2 (3), 1-28.

Earman, J., 2003. Tracking down gauge: an ode to the constrained Hamiltonian formalism. In: Brading, K., Castellani, E. (Eds.), Symmetries in physics. Cambridge University Press, pp. 150-162.

Geroch, R., Feb. 1970. Domain of Dependence. Journal of Mathematical Physics 11, 437-449.

Giulini, D., Apr. 2009. The Superspace of geometrodynamics. General Relativity and Gravitation 41, 785-815.

Gomes, H., Gryb, S., Koslowski, T., Feb. 2011. Einstein gravity as a 3D conformally invariant theory. Classical and Quantum Gravity 28 (4), 045005-+.

Gotay, M., Nester, J., Hinds, G., 1978. Presymplectic manifolds and the DiracBergmann theory of constraints. Journal of Mathematical Physics 19, 23882399.

Henneaux, M., Teitelboim, C., 1992. Quantization of gauge systems. Princeton University Press.

Hojman, S. A., Kuchař, K., Teitelboim, C., Jan. 1976. Geometrodynamics regained. Annals of Physics 96, 88-135.

Isham, C., 1992. Canonical quantum gravity and the problem of time. Arxiv preprint gr-qc.

URL http://arxiv.org/abs/grqc/9210011

Isham, C., Kuchař, K., 1985. Representations of spacetime diffeomorphisms. II. Canonical geometrodynamics. Annals of Physics 164 (2), 316 - 333.

URL http://www.sciencedirect.com/science/article/pii/0003491685900193

Kuchař, K., 1991. The problem of time in canonical quantization of relativistic systems. In: Ashtekar, A., Stachel, J. (Eds.), Conceptual Problems of Quantum Gravity. Boston University Press, p. 141.

Kuchař, K., 1992. Time and interpretations of quantum gravity. In: 4th Canadian Conference on General Relativity .... World Scientific Singapore.

Lewis, D., 1983. Individuation by acquaintance and by stipulation. The Philosophical Review 92, 3-32. 
Lusanna, L., 08 2011. Canonical gravity and relativistic metrology: from clock synchronization to dark matter as a relativistic inertial effect.

URL http://arxiv.org/abs/1108.3224v1

Mach, E., 1960. The Science of Mechanics: A Critical and Historical Account of its Development. Trans. McCormack, T. J. Open Court Publishing Company, LaSalle, Illinois.

Mittelstaedt, P., 1976. Der Zeitbegriff in der Physik. B.I.-Wissenschaftsverlag, Mannheim, Germany.

Pons, J., Salisbury, D., Shepley, L., 1997. Gauge transformations in the Lagrangian and Hamiltonian formalisms of generally covariant theories. Phys. Rev. D 55 (2), 658-668.

Pons, J., Salisbury, D., Sundermeyer, K. A., 2010. Observables in classical canonical gravity: folklore demystified. Journal of Physics A: Mathematical and General 222 (12018).

Pooley, O., 2001. Relationism Rehabilitated? II: Relativity. URL http://philsci-archive.pitt.edu/221/

Rickles, D., 2007. Symmetry, Structure, and Spacetime. Vol. 3 of Philossphy and Foundations of Physics. Elsevier.

Rovelli, C., Oct. 1990. Quantum mechanics without time: A model. Phys. Rev. D 42, 2638-2646.

Rovelli, C., 1991. Time in quantum gravity: An hypothesis. Phys. Rev. D 43, 442 .

Rovelli, C., 2002. Partial observables. Phys. Rev. D 65, 124013.

Rovelli, C., 2004. Quantum Gravity. Cambridge University Press, Cambridge.

Saunders, S., 2003. Physics and Leibniz's Principles. In: Brading, K., Castellani, E. (Eds.), Symmetries in physics. Cambridge University Press, pp. 289-307.

Smeenk, C., Wuthrich, C., October 2009. Time travel and time machines. Forthcoming in C. Callender (ed.), Oxford Handbook of Time, Oxford University Press.

URL http://philsci-archive.pitt.edu/4915/

Souriau, J., 1997. Structure of dynamical systems: a symplectic view of physics. Birkhauser.

Teitelboim, C., 1973. How commutators of constraints reflect the spacetime structure. Annals of Physics 79 (2), 542 - 557.

URL http://www.sciencedirect.com/science/article/pii/0003491673900961 
Thébault, K. P. Y., 2011a. Quantisation, representation and reduction; how should we interpret the quantum hamiltonian constraints of canonical gravity? Symmetry 3, 134.

Thébault, K. P. Y., May 2011b. Symplectic reduction and the problem of time in nonrelativistic mechanics.

URL http://philsci-archive.pitt.edu/8623/

Thiemann, T., 2006. The phoenix project: master constraint programme for loop quantum gravity. Classical and Quantum Gravity 23, 2211.

Thiemann, T., 2007. Modern canonical quantum general relativity. Cambridge University Press.

Weyl, H., 1922. Space-time-matter. Dover, New York, NY, translation by Brose, Henry L from the original German text into English.

Wheeler, J. A., 1968. Superspace and the Nature of Quantum Geometrodynamics. In: Wheeler, J. A., De Witt, C. M. (Eds.), Lectures in Mathematics and Physics. Benjamin.

York, Jr., J. W., Apr. 1973. Conformally invariant orthogonal decomposition of symmetric tensors on Riemannian manifolds and the initial-value problem of general relativity. Journal of Mathematical Physics 14, 456-464. 